\title{
1 A fungal member of the Arabidopsis thaliana phyllosphere antagonizes Albugo laibachii via a secreted lysozyme
}

Katharina Eitzen ${ }^{1,2}$, Priyamedha Sengupta ${ }^{1}$, Samuel Kroll ${ }^{2}$, Eric Kemen ${ }^{\star 2,3}$, Gunther Doehlemann*1

${ }^{1}$ Institute for Plant Sciences and Cluster of Excellence on Plant Sciences (CEPLAS),

7 University of Cologne, Center for Molecular Biosciences, Zuelpicher Str. 47a, 50674

8 Cologne, Germany.

$9{ }^{2}$ Max Planck Institute for Plant Breeding Research, Carl-von-Linne-Weg 10, 50829

10 Köln, Germany.

${ }^{3}$ Department of Microbial Interactions, IMIT/ZMBP, University of Tübingen, Tübingen,

12 Germany.

* correspondence to:

17 Eric Kemen, eric.kemen@uni-tuebingen.de,

Gunther Doehlemann, g.doehlemann@uni-koeln.de

Short title: Microbial antagonism suppressing Albugo laibachii infection

Key words: microbial antagonism, plant pathogen, Albugo laibachii, Ustilaginales, 


\section{Abstract}

28 Plants are not only challenged by pathogenic organisms, but also colonized by commensal microbes. The network of interactions these microbes establish with their host and amongst each other is suggested to contribute to the immune responses of plants against pathogens. In wild Arabidopsis thaliana populations, the oomycete pathogen Albugo laibachii has been shown to play an influential role in structuring the leaf phyllosphere. We show that the epiphytic yeast Moesziomyces bullatus ex Albugo on Arabidopsis, a close relative of pathogenic smut fungi, is an antagonistic member of the $A$. thaliana phyllosphere, which reduces infection of $A$. thaliana by $A$. laibachii. Combination of transcriptome analysis, reverse genetics and protein characterization identified a GH25 hydrolase with lysozyme activity as the major effector of this microbial antagonism. Our findings broaden the understanding of microbial interactions within the phyllosphere, provide insights into the evolution of epiphytic basidiomycete yeasts and pave the way for the development of novel biocontrol strategies.

\section{Introduction}

Plants are colonized by a wide range of microorganisms. While some microbes enter the plant and establish endophytic interactions with a broad range of outcomes from beneficial to pathogenic, plant surfaces harbor a large variety of microbial organisms. Recent research has focused largely on the importance of the rhizosphere microbiota in nutrient acquisition, protection from pathogens, and boosting overall plant growth and development (1-3). However, the above ground parts of the plant including the phyllosphere are colonized by diverse groups of microbes that also assist in plant protection and immunity $(4,5)$. The environment has a major impact on the microbial communities of the leaf surface, ultimately influencing their interactions with the host (6).

Scale-free network analysis was performed with the leaf microbial population of Arabidopsis thaliana (7). The majority of the interactions between kingdoms, e.g. fungi and bacteria, were found to be negative, consistent with the fact that rather the antagonistic interactions stabilize a microbial community

(8). Phyllosphere network 
57 influential microbes which have severe effects on the community structure. The major

58 hub microbe in the $A$. thaliana phyllosphere is the oomycete Albugo laibachii, which is a pathogenic symbiont biotrophic of Arabidopsis (7). This pathogen has been shown to significantly reduce the bacterial diversity of epiphytic and endophytic leaf habitats. Since bacteria generally comprise a large proportion of the phyllosphere microbiome (9), phylogenetic profiling of $A$. thaliana was also directed towards identifying a small group of bacteria that frequently colonize $A$. thaliana leaves. The analysis helped to develop a synthetic community of bacteria for experiments in gnotobiotic plants. Besides bacteria and oomycetes, the microbiota of the $A$. thaliana leaf also comprises a broad range of fungi. Among those fungi, basidiomycete yeasts are frequently found and the most frequent ones are the epiphytic basidiomycete genus Dioszegia (7), as well as an anamorphic yeast associated with $A$. laibachii infection, classified as Pseudozyma. sp. and belonging to Ustilaginales. This order includes many pathogens of important crop plants, for example corn smut and loose smut of oats, barley and wheat are caused by Ustilago maydis, $U$. avenae, $U$. nuda and $U$. tritici, respectively. Generally, pathogenic development of smut fungi is linked with sexual recombination and plant infection is only initiated upon mating when two haploid sporidia form a dikaryotic filament (10). Ustilaginales Pseudozyma sp. yeasts, however, are not known to be pathogenic. While they are found in anamorphic stage, they epiphytically colonize a wide range of habitats via where an infrequent sexual recombination might occur (11). Phylogenetic reconstruction (12) showed that the smut pathogen of millet, Moesziomyces bullatus and four species of Pseudozyma, namely $P$. antarctica, $P$. aphidis, $P$. parantarctica and $P$. rugulosa form a monophyletic group. The latter does represent anamorphic and culturable stages of $M$. bullatus and, hence, can be grouped to this genus. Moesziomyces strains have been reported in a number of cases to act as microbial antagonists. A strain formerly classified as Pseudozyma aphidis (now Moesziomyces bullatus) inhibited

Xanthomonas campestris pv. vesicatoria, $X$. campestris pv. campestris,

Pseudomonas syringae pv. tomato, Clavibacter michiganensis, Erwinia amylovora, and Agrobacterium tumefaciens in-vitro and also led to the activation of induced defense responses in tomato against the 
Podosphaera xanthii (14). Pseudozyma churashimaensis was reported to induce

systemic defense in pepper plants against $X$. axonopodis, Cucumber mosaic virus, Pepper mottle virus, Pepper mild mottle virus, and broad bean wilt virus (15). In the present study, we explored the antagonistic potential of an anamorphic Ustilaginales yeast within the leaf microbial community of $A$. thaliana. We show that Moesziomyces bullatus ex Albugo on Arabidopsis (which will be referred to as MbA, from further on in this paper) prevents infection by the oomycete pathogen $A$. laibachii and identified fungal candidate genes that were upregulated in the presence of A. laibachii, when both the microbes were co-inoculated in the host plant. A knockout mutant of one of the candidates, which belongs to the glycoside hydrolase - family 25 (GH25), was found to lose its antagonistic abilities towards $A$. laibachii, providing mechanistic insights into fungal-oomycete antagonism within the phyllosphere microbiota. Functional characterization of GH25 will be an important step towards establishing $M b A$ as a suitable biocontrol agent.

\section{Results}

104 In a previous study we isolated a basidiomycetous yeast from Arabidopsis thaliana 105 leaves infected with the causal agent of white rust, Albugo laibachii (7). This yeast was 106 tightly associated with $A$. laibachii spore propagation. Even after years of subculturing in 107 the lab and re-inoculation of plants with frozen stocks of $A$. laibachii isolate Nc14, this yeast remained highly abundant in spore isolates. Phylogenetic analyses based on fungal ITS-sequencing identified the yeast as Pseudozyma sp. Those yeasts can be

110 found across the family of Ustilaginaceae, being closely related to pathogens of 111 monocots like maize, barley, sugarcane or sorghum (Figure 1A and (16)). Microscopic 112 analyses verified the morphological similarity between the putative Pseudozyma sp. and 113 the Ustilaginaceous pathogen Ustilago maydis, the causal agent of corn smut (Figure 114 1B; (16)). Based on phylogenetic similarity to the pathogenic smut 115 Moesziomyces bullatus which infects millet, several anamorphic Pseudozyma isolates 116 were suggested to be renamed and grouped to M. bullatus (12). Since the Pseudozyma 117 sp. that was isolated from $A$. laibachii spores groups into the same cluster, we classified 
118 this newly identified species as MbA (Moesziomyces bullatus ex Albugo on 119 Arabidopsis).

121 Based on the identification of $M b A$ as heaving a significant effect on bacterial diversity 122 in the Arabidopsis phyllosphere, we tested its interaction with 30 bacterial strains from 12317 different species of a synthetic bacterial community (SynCom, Table S1) of 124 Arabidopsis leaves in one-to-one plate assays. This experiment identified seven strains 125 being inhibited by Moesziomyces, as indicated by halo formation after 7 days of co126 cultivation (Supplementary Figure S1). Interestingly, this inhibition was not seen when 127 the pathogenic smut fungus $U$. maydis was co-cultivated with the bacteria, indicating a 128 specific inhibition of the bacteria by $M b A$ (Supplementary Figure S1).

129 The primary hub microbe in the Arabidopsis phyllosphere was found to be the pathogenic oomycete $A$. laibachii, which was isolated in direct association with Moesziomyces (7). To test if both species interfere with each other, we deployed a gnotobiotic plate system and quantified $A$. laibachii infection symptoms on Arabidopsis. In control experiments, spray inoculation of only $A$. laibachii spores on Arabidopsis leaves led to about 33\% infected leaves at 14 dpi (Figure 2). When the bacterial SynCom was pre-inoculated on leaves two days before $A$. laibachii spores a significant reduction of $A$. laibachii infection by about $50 \%$ was observed (Figure 2). However, if Moesziomyces was pre-inoculated with the bacterial SynCom, $A$. laibachii spore production was almost completely abolished. Similarly, the pre-inoculation of only MbA resulted in an almost complete loss of $A$. laibachii infection, independently of the presence of a bacterial community (Figure 2). The antagonistic effect of $M b A$ towards $A$. laibachii was further confirmed using Trypan blue staining of $A$. laibachii infected $A$. thaliana leaves. A. laibachii forms long, branching filaments on Arabidopsis leaves at 15 dpi. Contrary, in presence of $M b A$, we observed mostly zoospores forming either no or very short hyphae, while further colonization of the leaf with long, branching was not observed (Supplementary figure S2B). Together, of findings demonstrates that $M b A$ holds a strong antagonistic activity towards $A$. laibachii, resulting in efficient biocontrol of 
148

149

150

151

152

153

154

155

156

157

158

159

160

161

162

163

164

165

166

167

168

169

170

171

172

173

174

175

176

177

microbial community. However, despite, several reports of the basidiomycete yeasts acting as antagonists, genomic analysis of the said group is rather limited. We therefore sequenced the genome of $M b A$ and established molecular tools allowing functional genetic approaches.

\section{The genome of $M b A$.}

Genome sequence of $M b A$ was analyzed by Single Molecule Real-Time sequencing (Pacific Biosciences, Menlo Park, CA), which lead to 69674 mapped reads with an accuracy of $87.3 \%$ and $8596 \mathrm{bp}$ sub-read length. Sequence assembly using the HGAPpipeline (Pacific Biosciences) resulted in 31 Contigs with a $\mathrm{N}_{50}$ Contig Length of $705 \mathrm{~kb}$. The total length of all contigs results in a predicted genome size of 18.3Mb (Table 1). Gene prediction for the $M b A$ genome with Augustus (17) identified 6653 protein coding genes, of which 559 carry a secretion signal. Out of these 559, 380 are predicted to be secreted extracellularly (i.e. they do not carry membrane domains or cell-wall anchors) (Table 1). The small genome size and high number of coding genes results in a highly compact genome structure with only small intergenic regions. These are features similarly found in several pathogenic smut fungi such as $U$. maydis and $S$. reilianum (Table 1). Remarkably, both $M b A$ and Anthracocystis flocculosa, which is another anamorphic and apathogenic yeast, show a similarly high rate of introns, while the pathogenic smut fungi have a significantly lower intron frequency (Table 1).

To gain better insight in the genome organization of $M b A$, we compared its structure with the $U$. maydis genome, which serves as a manually annotated high-quality reference genome for smut fungi (18). Out of the $31 \mathrm{MbA}$ contigs, 21 show telomeric structures and a high synteny to chromosomes of $U$. maydis, with three of them displaying major events of chromosomal recombination (Figure $3 \mathrm{~A}$ ). Interestingly, the Moesziomyces contig 2, on which also homologs to pathogenic loci like the $U$. maydis virulence cluster $2 \mathrm{~A}$ (18) can be found, contains parts of three different $U$. maydis chromosomes (Chr. 2, 5, 20) (Supplementary figure S3). The second recombination event on contig 6 affects the $U$. maydis leaf-specific virulence factor see1, which is required for tumor formation (19). This recombination event is also found in the genome 
178 of the maize head smut $S$. reilianum, wherein the $U$. maydis chromosomes 5 and 20 179 recombined in the promoter region of the see1 gene (Figure 3B). In this respect it should be noted that $S$. reilianum, although infecting the same host, does not produce leaf tumors as $U$. maydis does (20).

Also the third major recombination event, affecting $M b A$ contig 8, changes the genomic context genes encoding essential virulence factors in $U$. maydis (stp1 \& pit1/2), as well as the A mating type locus, which is important for pheromone perception and recognition of mating partners (21). Based on the strong antibiotic activities of $M b A$, we mined the genome of $M b A$ for the presence of secondary metabolite gene clusters. Using AntiSMASH, we were able to predict 13 of such clusters, of which three can be assigned to terpene synthesis, three contain non-ribosomal peptide synthases and one cluster has a polyketide synthetase as backbone genes (Supplementary Figure S4A). Interestingly, the secondary metabolite cluster that is involved in the production of the antimicrobial metabolite ustilagic acid in other Ustilaginomycetes, is absent in $M b A$ (Supplementary Figure S4B). On the contrary, we could identify three MbA specific metabolite clusters which could potentially be involved in the antibacterial activity of MbA (Supplementary Figure S4C).

A previous genome comparison of the related Ustilaginales yeast $A$. flocculosa with $U$. maydis concluded that this anamorphic strain had lost most of its effector genes, reflecting the absence of a pathogenic stage in this organism (22). In contrast, $M b A$ contains 1:1 homologs of several known effectors with a known virulence function in $U$. maydis (Table 2). We previously found that Moesziomyces sp. possess functional homologues of the pep1 gene, a core virulence effector of $U$. maydis (23), suggesting that such anamorphic yeasts have the potential to form infectious filamentous structures by means of sexual reproduction (11). To assess the potential virulence activity of $M b A$ effector homologs, we expressed the homolog of the $U$. maydis core effector Pep1 in an U. maydis pep1 deletion strain (SG200401987). This resulted in complete restoration of $U$. maydis virulence, demonstrating that, when ectopically expressed, MbApep1 encodes a functional effector (Supplementary Figure S5). 
A hallmark of the $U$. maydis genome structure is the presence of large clusters with effector genes, the expression of which is only induced during plant infection (18). To assess the presence of potential virulence clusters in $M b A$, we compared all $U$. maydis effector gene clusters to the $M b A$ genome, based on homology. This revealed that the twelve major effector clusters of $U$. maydis are present in $M b A$. However, while many of the clustered effector genes are duplicated in pathogenic smut fungi, $M b A$ carries only a single copy of each effector gene. This results in the presence of "short" versions of the $U$. maydis gene effector clusters (Supplementary Figure S6). This gets particularly obvious for the biggest and most intensively studied virulence cluster of smut fungi, the effector cluster 19A $(20,24,25)$. In $M b A$ only three out of the 24 effector genes present in $U$. maydis are conserved in this cluster (Figure 4). Interestingly, some anamorphic yeasts like Kalmanozyma brasiliensis and $A$. flocculosa completely lost virulence clusters, while another non-pathogenic member of the Ustilaginales, Pseudozyma hubeiensis, shows an almost complete set of effectors when compared to U. maydis (Figure 4).

\section{Genetic characterization of $M b A$}

To perform reverse genetics in $M b A$, we established a genetic transformation system based on protoplast preparation and PEG-mediated DNA transfer. In preliminary transformation assays, we expressed a cytosolic GFP reporter-gene under control of the constitutive o2tef-Promoter (Figure 5A). For the generation of knockout strains, a split marker approach was used to avoid ectopic integrations (Figure 5B). To allow generation of multiple knockouts, we used a selection marker-recycling system (pFLPexpC) which allows selection marker excision at each transformation round (26).

We decided to apply the transformation system to study the MbA mating type loci in more detail. Although phylogenetically closely related to $U$. hordei, which has a bi-polar mating system, $M b A$ owns a tetrapolar mating system whereby both mating type loci are physically not linked. This situation is similar to the mating type structure in the pathogenic smut $U$. maydis (Figure $5 \mathrm{~A}$ ). The a-locus, which encodes a pheromone receptor system that is required for sensing and fusion of compatible cells, is located on 
237 contig 6 . The $b$-locus can be found on contig 1. This multiallelic mating locus contains 238 two genes (b-East and $b$-West), which code for a pair of homeodomain transcription factors. Upon mating of compatible cells, pathogenic and sexual development are triggered by a heterodimeric bE/bW complex (10). Since the $M b A$ genome is completely equipped with mating type genes, we first deployed a screen for potential mating partners. To this end, we screened wild $M$. bullatus isolates to find a suitable mating partner, but we could not observe any mating event (Supplementary Figure S7). To test if $M b A$ is able to undergo pathogenic differentiation in the absence of mating, we generated a self-compatible strain (CB1) which carries compatible b-mating alleles: to construct the CB1 strain, we used compatible alleles of the $b$-East and $b$-West genes of the barley smut $U$. hordei, a pathogen which is the phylogenetically most closely related to $M b A$ and amenable to reverse genetics. The native $M b A$ locus was replaced by the compatible $U$. hordei $b$-East and $b$-West gene alleles via homologous recombination (Figure 6B).

Incubation of the MbA CB1 on charcoal plates led to the formation of aerial hyphae with 252 the characteristic fluffy phenotype of filamentous strains like the self-compatible, 253 solopathogenic U. maydis SG200 strain (Figure 6C). A second established method to induce filament formation in smuts is on hydrophobic parafilm (27). Quantification after 18 hours incubation of $M b A C B 1$ on parafilm resulted in the formation of filaments 256 comparable to those of the $U$. maydis SG200 strain (Figure 6D). While about 17\% of $257 \mathrm{MbA}$ wild type cells showed filaments, the CB1 strain with compatible b-genes showed $25838 \%$ filamentous growth.

259 Formation of appressoria is a hallmark of pathogenic development in smut fungi (27). 260 While the switch from yeast-like growth to filamentous development is the first step in 261 the pathogenic development of smut fungi, host penetration is accompanied by the 262 formation of a terminal swelling of infectious hyphae, termed "appressoria". Induction of 263 appressoria-formation in vitro can be induced by adding $100 \mu \mathrm{M}$ of the cutin monomer 264 16-Hydroxyhexadecanoic acid (HDD) to the fungal cells prior to cell spraying onto a 265 hydrophobic surface (27). In absence of HDD, only about 8\% of the U. maydis SG200 266 cells and $14 \%$ of the MbA cells formed appressoria on parafilm 24 hours after spraying 267 (Figure 6E). Addition of $100 \mu \mathrm{M}$ HDD resulted in a significant induction of appressoria in 
268 both $U$. maydis and $M b A$, demonstrating that $M b A$ does hold the genetic repertoire to 269 form infection structures in-vitro. Together, the analysis of the recombinant CB1 strain 270 indicates that $M b A$ can sense pathogenesis-related surface cues and produce 271 penetration structures to a similar level as that seen for the pathogenic model organism 272 U. maydis.

273

274

275

276

277

278

279

280

281

282

283

284

285

286

287

288

289

290

291

292

293

294

295

296

297

298

\section{Identification of microbe-microbe effector genes by RNA-Seq}

To study the transcriptomic response of $M b A$ to different biotic interactions, RNA sequencing was performed. The $M b A$ transcriptome was profiled in five different conditions (Figure 7A; cells in axenic culture versus cells on-planta, on-planta + SynCom, on-planta $+A$. laibachii, on-planta + SynCom $+A$. laibachii). Inoculations of $A$. thaliana leaves were performed as described above for $A$. laibachii infection assays (Figure 7A). For MbA RNA preparation, the epiphytic microbes were peeled from the plant tissue by using liquid latex (see methods section for details).

The libraries of the 15 samples (five conditions in three biological replicates each) were generated by using a poly-A enrichment and sequenced on an Illumina HiSeq4000 platform. The paired end reads were mapped to the MbA genome by using Tophat2 (28). The analysis revealed that $M b A$ cells on $A$. thaliana leaves (on-planta) downregulated 1300 and upregulated 1580 genes compared to cells in axenic culture (Figure 7B). Differentially expressed genes were determined with the "limma"-package in R on "voom" (Supplementary Figure S8) using a False discovery rate threshold of 0.05 and $\log 2 \mathrm{FC}>0$. A GO-terms analysis revealed that, among the downregulated genes, $50 \%$ were associated with primary metabolism (Supplementary Figure S9). In the two conditions in which $A$. laibachii was present, we observed upregulation of 801 genes. Among these genes, 411 genes were specific to co-incubation of $M b A$ with $A$. laibachii and SynCom while 174 were specific to incubation with $A$. laibachii only. A set of 216 genes was shared in both conditions (Figure 7B).

In presence of $A$. laibachii, mainly metabolism- and translation-dependent genes were upregulated, which might indicate that $M b A$ can access a new nutrient source in presence of $A$. laibachii (Supplementary Figure S9). Among all $A$. laibachii-induced $M b A$ genes, 25 genes encode proteins carrying a secretion signal peptide and having 
299 no predicted transmembrane domain (Figure 7C). After excluding proteins being 300 predicted to be located in intracellular organelles, nine candidate genes remained as potential microbe-microbe dependent effectors, i.e. $M b A$ genes which are induced by $A$. laibachii, show no or low expression in axenic culture and encode for putative secreted 303 proteins (Figure 7C). Interestingly, four of these genes encode putative glycoside hydrolases. Furthermore, two genes encode putative peptidases, one gene likely encodes an alkaline phosphatase and two encode uncharacterized proteins (Figure 306 7C).

307 To directly test the eventual antagonistic function of those genes towards $A$. laibachii, we selected the two predicted glycoside hydrolases-encoding genes g5 \& g2490 (GH43 \& $\mathrm{GH} 25)$ and the gene encoding the uncharacterized protein $g 5755$ for gene deletion in $M b A$. The respective mutant strains were tested in stress assays to assess, whether the gene deletions resulted in general growth defects. Wild type and mutant MbA strains were exposed to different stress conditions including osmotic stress (sorbitol, $\mathrm{NaCl}$ ), cell wall stress (calcofluor, congored) and oxidative stress $\left(\mathrm{H}_{2} \mathrm{O}_{2}\right)$. Overall, in none of the tested conditions we observed a growth defect of the deletion mutants in comparison to wild type MbA (Supplementary Figure S10). To test an eventual impact of the deleted genes in the antagonism of the two microbes, the MbA deletion strains were each preinoculated on $A$. thaliana leaves prior to $A$. laibachii infection. Deletion of $g 5$ resulted in a significant but yet marginal increase of $A$. laibachii disease symptoms, while deletion 319 of $g 5755$ had no effect on A. laibachii. We therefore considered these two genes being not important for the antagonism of $M b A$ towards $A$. laibachii. Strikingly, the $M b A \Delta g 2490$ strain almost completely lost its biocontrol activity towards $A$. laibachii.

322 This phenotype was reproduced by two independents $g 2490$ deletion strains (Figure $3238 \mathrm{~A}$ ). To check if this dramatic loss of microbial antagonism is specific to the deletion of 324 g2490, in-locus genetic complementation of strain $\Delta g 2490 \_1$ was performed via 325 homologous recombination. The resulting strain $M b A \Delta g 2490 / c o m p /$ regained the ability 326 to suppress $A$. laibachii infection, confirming that the observed phenotype specifically 327 resulted from the deletion of the g2490 gene (Figure 8B). Together, these results 328 demonstrate that the biocontrol of the pathogenic oomycete $A$. laibachii by the basidiomycete yeast $M b A$ is determined by the secretion of a previously 
uncharacterized GH25 enzyme, which is transcriptionally activated specifically when both microbes are co-colonizing the $A$. thaliana leaf surface.

\section{Functional characterization of the secreted MbA hydrolase}

To characterize the protein function of the GH25 encoded by MbA g2490, we were using Pichia pastoris for heterologous expression. The recombinant protein was tagged with polyhistidine tag for Ni-NTA affinity purification. The purified protein was detected at an expected size of 27kDa (Supplementary Figure S11A). In addition, via site directed mutagenesis a mutated version of the protein was generated, carrying a single amino exchange at the predicted active site (GH25_D124E). Both active and mutated versions of the GH25 hydrolase were subjected to a quantitative lysozyme activity assay using fluorogenic substrate Micrococcus lysodeikticus with commercial Hen egg-white lysozyme as a control. We noticed a concentration dependent increase in relative fluorescence unit (RFU)/min for the active GH25 in molar concentrations from $2 \mathrm{uM}$ to 10uM. Whereas, for similar concentrations, mutated GH25 (GH25mut) showed no significant increase in RFU/min compared to the active version. Commercial HEWL showed a steady increase in RFU/min from 1uM to $5.5 \mathrm{uM}$ concentrations (Figure 8C; Supplementary Figure S11C). Thus, the recombinant protein represents a functional GH25 hydrolase with a lysozyme activity.

To test for a direct function of the GH25 lysozyme, we treated $A$. laibachii-infected Arabidopsis plants with the recombinant protein. The impact of treatment of $A$. laibachii infection was quantified by quantitative PCR to determine the relative $A$. laibachii biomass on Arabidopsis in response to GH25. Strikingly, we observed a significant reduction of $A$. laibachii colonization in leaves treated with the active GH25 lysozyme, while the mutated enzyme GH25_D124E did not significantly influence infection ( $p$ value of $<0.0001$ and an R-squared value of 98.88\%) (Figure 8D). Overall, treatment with the $\mathrm{GH} 25$ lysozyme reduced the amount of $A$. laibachii to about $50 \%$.

\section{Discussion}

Healthy plants in natural habitats are extensively colonized by microbes, therefore it has been hypothesized that the immune system and the microbiota may instruct each other 
361 beyond the simple co-evolutionary arms race between plants and pathogens (29).

362 Community members as individuals or in a community context have been reported to confer extended immune functions to their plant host. Root endophytic bacteria for example were found to protect $A$. thaliana and stabilize the microbial community by competing with filamentous eukaryotes (30). A large inhibitory interaction network was found in the leaf microbiome of $A$. thaliana and genome mining was used to identify over 1000 predicted natural product biosynthetic gene clusters (BGCs) (31). In addition, the bacterium Brevibacillus sp. leaf 182 isolate was found to inhibit half of the 200 strains isolated from $A$. thaliana phyllosphere. Further analysis revealed that Brevibacillus sp. leaf 182 produces a trans-acyltransferase polyketide synthase-derived antibiotic, macrobrevin along with other putative polyketide synthases (31).

372 In this study, we describe the role of the basidiomycete yeast $M b A$, which we previously 373 co-isolated with the oomycete pathogen $A$. laibachii and now characterized as an 374 antagonistic driver in the $A$. thaliana phyllosphere. A. laibachii inhibits in-vitro growth of seven members of a bacterial leaf SynCom and, most strikingly, strongly suppresses disease progression and reproduction of the pathogenic oomycete $A$. laibachii on $A$.

377 thaliana. $M b A$ is a member of the Ustilaginales, which had previously been classified into the group of pathogenic smut fungi of the Moesziomyces bullatus species (11). Our genome analysis identified the anamorphic yeasts $M$. rugulosus, $M$. aphidis and $M$. antarcticus, which had previously been classified as "Pseudozyma spec.", as the closest relatives of $M b A$. Anamorphic Ustilaginales yeasts are long known and have been used for biotechnological applications and also biocontrol (32). Mannosylerythritol lipids produced by $M$. antarcticus are known to act as biosurfactants and are of great interest 384 for pharmaceutical applications $(33,34)$. Glycolipids like flocculosin produced by $A$. flocculosa or ustilagic acid characterized in the smut fungus $U$. maydis, have antifungal activity. Those compounds destabilize the membrane of different fungi and thus serve as biocontrol agents against powdery mildews or grey mold (35-37) We identified 13 potential secondary metabolite gene clusters in MbA, including nonribosomal peptide synthases and polyketide synthetase. Interaction among microbes within the same habitat is believed to have given rise to a variety of secondary 
392 activate an otherwise silent polyketide synthase gene cluster, $\operatorname{fgn} A$, in Aspergillus 393 fumigatus. The resultant compound proved to be a potent fungal metabolite that 394 inhibited the germination of $S$. rapamycinicus spores (40). Therefore, secondary 395 metabolite gene clusters and their corresponding products may confer a competitive 396 advantage to fungi over the bacteria that reside in the same environment.

397 What is still under debate is the relation of anamorphic yeasts with the related 398 pathogenic smuts. Many smut fungi, including the model species $U$. maydis are 399 dimorphic organisms. In their saprophytic phase they grow as haploid non-pathogenic 400 yeast cells. Only on appropriate host surfaces, haploid cells switch to filamentous 401 growth and expression of pathogenicity-related genes is only activated upon mating in 402 the filamentous dikaryon. A prime prerequisite for pathogenic development is therefore 403 the ability of mating $(41,42)$. Our genome analysis identified a tetrapolar mating system 404 with a complete set of mating genes in MbA. Looking more closely on the phylogeny of 405 different mating genes it appears that all sequenced Moesziomyces strains have the 406 same pheromone receptor type (Supplementary Figure S12). Together with our 407 unsuccessful mating assays, this suggests that all sequenced strains of this species 408 have the same mating type and, therefore, are unable to mate. Mating type bias after 409 spore germination was reported for Ustilago bromivora, which leads to a haplo-lethal 410 allele linked to the MAT-2 locus (43). In this case, an intratetrad mating event rescues 411 pathogenicity in nature as the second mating partner is not viable after spore 412 germination. Together with the observation that anamorphic Moesziomyces yeasts are 413 ubiquitous in nature, one could hypothesize that these fungi are highly competitive in 414 their haploid form and antagonism might have led to the selection of one viable mating 415 type. This eventually adapted to the epiphytic life style.

416 Transcriptome analysis showed that epiphytic growth of $M b A$ on $A$. thaliana leads to 417 massive transcriptional changes particularly in primary metabolism, which might reflect 418 adaptation to the nutritional situation on the plant surface. Moreover, MbA showed 419 specific transcriptional responses to a bacterial community, as well as to $A$. laibachii 420 when being co-inoculated on plant leaves. Presence of $A$. laibachii resulted in the 421 induction of primary metabolism and biosynthesis pathways, which might reflect enhanced growth of $M b A$ in the presence of $A$. laibachii. 
423 A set of $M b A$ genes encoding secreted hydrolases was induced by $A$. laibachii and one

424 of these genes which encodes a putative GH25 hydrolase with similarity to Chalaropsis 425 type lysozymes appeared to be essential for the biocontrol of $A$. laibachii. Initially 426 discovered in the fungi Chalaropsis sp., this group of proteins is largely present in 427 bacteria as well as phages for example the germination specific muramidase from 428 Clostridium perfringens S40 (44). The bacterial muramidase, cellosyl from 429 Streptomyces coelicolor (45) also belongs to the Chalaropsistype of lysozyme. These 430 proteins are proposed to cleave the $\beta$-1,4-glycosidic bond between $\mathrm{N}$-acetylmuramic 431 acid (NAM) and N-acetylglucosamine (NAG) in the bacterial peptidoglycan. Specifically, 432 the $\beta-1,4-\mathrm{N}, 6-\mathrm{O}-$ diacetylmuramidase activity allows the Chalaropsis type lysozyme to 433 degrade the cell wall of Staphylococcus aureus, in contrast to the commercially 434 available Hen egg-white lysozyme (HEWL) (45). Despite differences in structure and 435 molecular weight from HEWL, the GH25 of MbA has lysozyme activity against the gram 436 positive bacterium Micrococcus lysodeikticus in a fluorogenic assay. This highlights the 437 overall biochemical functionality of the recombinant glycoside hydrolase. The glycoside 438 Hydrolase 25 family is predicted to have an active site motif DXE which is highly 439 conserved across the fungal kingdom (Supplementary Figure S13). The structure of 440 glycoside hydrolase family 25 from Aspergillus fumigates was characterized and the 441 presence of $\mathrm{N}$-terminal signal peptide was considered to indicate an extracellular 442 secretion of the protein with possible antimicrobial properties (46). The role of the secreted hydrolase in the fungal kingdom is not completely explored yet. The presence 444 of such hydrolases has in many cases been hypothesized to be associated with 445 hyperparasitism of fungi parasitizing fungi (47) or oomycetes parasitizing oomycetes 446 (48). Our results might therefore indicate a cross kingdom hyperparasitism event 447 between a fungus and an oomycete. Previous work on microbial communities has 448 indicated that negative interactions stabilize microbial communities. Hyperparasitism is 449 such a negative interaction with a strong eco-evolutionary effect on pathogen-host 450 interactions and therefore on community stability (49). MbA might therefore regulate $A$. 451 laibachii infection and reduce disease severity. The qPCR evaluation of oomycete 452 biomass strongly points towards the idea that $A$. laibachii is a direct target of 453 antagonism for MBA. Since we observed reduced formation of $A$. laibachii in presence 
454 of $M b A$, we also tested if the GH25 lysozyme would suppress zoospore germination. 455 However, we could not detect a significant reduction of $A$. laibachii zoosporangia 456 germination upon treatment with active GH25 lysozyme (Supplementary figure 457 S14), suggesting that the GH25 lysozyme interferes with $A$. laibachii at a later stage of 458 infection. As $A$. laibachii has been shown to reduce microbial diversity (7), MbA might increase diversity through hyperparasitism of $A$. laibachii. At the same time this increased diversity might have caused the need for more secondary metabolites to evolve in the $M b A$ genome to defend against niche competitors. Through its close association with $A$. laibachii, $M b A$ could be a key regulator of the $A$. thaliana microbial diversity and therefore relevant for plant health beyond the regulation of $A$. laibachii 464 infection.

465 In conclusion, the secreted hydrolase we identified as main factor of $A$. laibachii inhibition has great potential to act as antimicrobial agent. The isolated compound is not only valuable per se in an ecological context. It can further lay the grounds for exploring other microbial bioactive compounds that mediate inter-species and inter-kingdom crosstalk. A main goal of our future studies will be to understand on the mechanistic level, how the GH-25 suppresses $A$. laibachii, and at which developmental step the oomycete infection is blocked. Since the GH-25 enzyme is well conserved amongst

472 Ustilaginales including pathogenic species, it will also be tempting to elucidate whether 473 the species-specific antagonism identified here is broadly conserved among 474 Ustilaginales fungi and oomycetes. We further will investigate potential responses by 475 the host plant and how this impacts $A$. laibachii growth upon MbA colonization. 476 Functional investigation of these interactions can provide meaningful insights as to why 477 certain yeasts prefer to colonize specific environments. At the same time, it will be worth 478 exploring how the basidiomycete yeasts influence the bacterial major colonizers of the 479 phyllosphere.

\section{Material and Methods}

$483 \mathrm{MbA}$ wildtype strain was isolated from $A$. laibachii infected $A$. thaliana leaves [7]. Wild- 
YEPSlight medium and maintained on Potato dextrose agar plates. King's B medium was used for culturing Syn Com bacterial members at 22 degrees. All the strains were grown in a rotary shaker at 200rpm. All the recipes for medium and solutions can be found in Supplementary Table S2. Stress assays for fungi: wildtype and mutant strains of $M b A$ grown to an optical density $(600 \mathrm{~nm})$ of $0.6-0.8$ were centrifuged at $3500 \mathrm{rpm}$ for 10 minutes and suspended in sterile water to reach an OD of 1.0. Next, a dilution series from $10^{0}$ to $10-4$ was prepared in sterile $\mathrm{H}_{2} \mathrm{O}$. In the end, $5 \mu \mathrm{l}$ of each dilution were spotted on CM plates supplemented with the indicated stress agents. The plates were incubated for 2 days at $22^{\circ} \mathrm{C}$. Confrontation assays: at first, $M b A$ and SynCom bacterial strains were grown to an O.D of 0.8-1. MbA cultures (10ul) were dropped in four quadrants of a Potato Dextrose Agar plate, previously spread with a bacterial culture. Plates were incubated for $2-4$ days at $22^{\circ} \mathrm{C}$.

Transformation of $M b A$ and plasmid construction for generation of knockout mutants

Fungal strains were grown in YEPSL at $22^{\circ} \mathrm{C}$ in a rotary shaker at $200 \mathrm{rpm}$ until an O.D. of 0.6 was reached and centrifuged for $15 \mathrm{mins}$ at $3500 \mathrm{rpm}$. The cells were washed in $20 \mathrm{ml}$ of SCS (Table S2), and further centrifuged for 10 minutes at $3000 \mathrm{rpm}$, before being treated with $3 \mathrm{ml} \mathrm{SCS}$ solution with $20 \mathrm{mg} / \mathrm{ml}$ of Glucanex (Lysing Enzyme from Trichoderma harzianum, \# L1412, Sigma). After 20 minutes of incubation at room temperature, as cell wall lysis was occurred, cold SCS was added to the mix and protoplasts spun down for 10 minutes at 2400rpm. They were then washed twice with SCS and resuspended with $10 \mathrm{ml} \mathrm{STC} \mathrm{(SupplementaryTable} \mathrm{S2)} \mathrm{to} \mathrm{be} \mathrm{centrifuged} \mathrm{at}$ 2000rpm for 10 minutes. Finally, the pellet was dissolved in $500 \mu \mathrm{ISTC}$, and stored in aliquots of $50 \mu \mathrm{l}$ at $-80^{\circ} \mathrm{C}$. $5 \mu \mathrm{g}$ of plasmid DNA along with $15 \mu \mathrm{g}$ Heparin was added to $50 \mu \mathrm{l}$ protoplasts. After incubation on ice for 10 minutes, STC/40\%PEG $(500 \mu \mathrm{l})$ was added to it and mixed gently by pipetting up and down; this step was followed by another 15 minutes on ice. The transformation mix was added to $10 \mathrm{ml}$ of molten regeneration (reg) agar and poured over a layer of already solidified reg agar containing appropriate antibiotic solution. For the bottom layer, we used $400 \mu \mathrm{g} / \mathrm{ml} \mathrm{Hygromycin/} 8$ $\mu \mathrm{g} / \mathrm{ml}$ Carboxin/ $300 \mu \mathrm{g} / \mathrm{ml}$ nourseothricin (NAT). 
516 Plasmids were cloned using Escherichia coli DH5a cells (Invitrogen, Karlsruhe,

517 Germany). Construction of deletion mutants was performed by homologous

518 recombination; the 5' and 3' flanking regions of the target genes were amplified and

519 ligated to an antibiotic resistance cassette (50). The ligated fragment was subsequently

520 transformed into $M b A$. Homologous integration of the target gene was verified via PCR

521 on the antibiotic resistant colonies. Oligonucleotide pairs for knockout generation and

522 verification can be found in Supplementary Table S3. PCR amplification was done using

523 Phusion@ DNA polymerase (Thermo Scientific, Bonn, Germany), following the

524 manufacturer's instructions, with $100 \mathrm{ng}$ of genomic DNA or cDNA as template. Nucleic

525 acids were purified from 1\% TAE agarose gels using Macherey-Nagel ${ }^{\mathrm{TM}}$ NucleoSpin $^{\mathrm{TM}}$

526 Gel and PCR Clean-up Kit.

527

528

Mating assay and generation of the self-compatible MbA strain CB1

529 Haploid strains of $M b A$ were grown in liquid cultures, mixed and drops arranged on PD-

530 plates with charcoal to induce filament formation. Plate with the haploid U. maydis

531 strains FB1 and FB2 and the solopathogenic strain SG200 served as internal control.

532 The complete b-locus of the solopathogenic $U$. hordei strain DS200 was amplified

533 (Figure S2) and inserted into the $M b A$ b-locus by homologous recombination. The strain

534 obtained, known as compatible b1 (CB1) was tested positive by amplification of the right

535 border and left border areas with primers specific for the genomic locus and for the

536 plasmid region. Additionally, two primers specific for the MbA $b$ Eand $b W$ genes were

537 chosen to amplify parts of the native locus. To induce filament and appressoria

538 formation in vitro we used a Moesziomyces YEPSL culture at $\mathrm{OD}_{600}$ 0.6-0.8. The cells

539 were diluted to an $\mathrm{OD}_{600}$ of 0.2 in $2 \%$ YEPSL (for appressoria formation $100 \mu \mathrm{M} 16$ -

540 hydroxyhexadecanoic acid (Sigma-Aldrich) or $1 \%$ ethanol was added) and sprayed the

541 yeast like cells on parafilm which mimics the hydrophobic plant surface. After 18h

542 incubation at $100 \%$ humidity the number of cells grown as filaments (or generating

543 appressoria) was determined relative to the total number of total cells by using a light

544 microscope. 


\section{Arabidopsis thaliana leaf infections and quantification of Albugo biomass}

\section{quantification by qPCR}

Sterilized Arabidopsis thaliana seeds were subjected to cold treatment for 7 days and sown on 1/2 strength Murashige Skoog (MS) medium (Supplementary Table S2). The MS plates are directly transferred to growth chambers having $22^{\circ} \mathrm{C}$ on a short-day period (8 $\mathrm{h}$ light) with (33-40\%) humidity and grown for 4 weeks before inoculation. Overnight liquid cultures of $M b A$ and SynCom bacterial strains were grown to an $O_{600}$ of 0.6. The cultures were spun down at 3500rpm for 10 minutes and the pellets dissolved in $\mathrm{MgCl}_{2} .500 \mu \mathrm{l}$ of each culture was evenly sprayed on three-week old $A$. thaliana seedlings using airbrush guns. Two days later, a spore solution of $A$. laibachii was then sprayed on the seedlings following the protocol of Ruhe et al. (51). Two weeks later, the disease symptoms on the leaves were scored as a percentage between infected and non-infected leaves.

4 weeks old $A$. thaliana seedlings on MS plates were sprayed with $A$. laibachii as a control and $\mathrm{GH} 25+$ A.laibachii and Mut_GH25+A.laibachii as treatments. After 10 days post infection (dpi), the seedlings were harvested, frozen in liquid nitrogen and kept at $80^{\circ} \mathrm{C}$. For DNA extraction, the frozen plant material was ground into a fine powder with mortar and pestle and treated with extraction buffer $(50 \mathrm{mM}$ Tris $\mathrm{pH} 8.0,200 \mathrm{mM}$ $\mathrm{NaCl}, 0.2 \mathrm{mM}$ ethylenediaminetetraacetic acid (EDTA), 0.5\% SDS, $0.1 \mathrm{mg} / \mathrm{ml}$ proteinase $\mathrm{K}$ (Sigma-Aldrich). This was followed centrifugation after the addition of one volume Phenol/Chloroform/Isoamylalkohol 25:24:1 (Roth). The top aqueous layer was removed and added to one volume of Isopropanol to precipitate the nucleic acids. DNA pellet obtained after centrifugation was washed with $70 \% \mathrm{EtOH}$ and finally dissolved in $50 \mathrm{ul}$ Nuclease-free water. For qPCR measurements; 10 ul of GoTaq ${ }^{\circledR}$ qPCR 2x Master Mix was used (Promega, Waltham, Madison, USA); 5ul of DNA ( 50ng); 1ul of forward and reverse primer $(10 \mu \mathrm{M})$ up to a total volume $20 \mu \mathrm{l}$. Samples were measured in triplicates in a CFX Connect real-time PCR detection system (Bio-Rad) following protocol of Ruhe et al. (2016)(51). Amount of $A$. laibachii DNA was quantified using the following oligonucleotide sequences, (A. thalianaEF1-a: 5'-AAGGAGGCTGCTGAGATGAA-3', 5'TGGTGGTCTCGAACTTCCAG-3'; Oomycete internal transcribed spacer (ITS) 5.8s: 5'ACTTTCAGCAGTGGATGTCTA-3', 5'-GATGACTCACTGAATTCTGCA-3'). Cq values 
577 obtained in case of the oomycete DNA amplification was normalized to $A$. thalaina DNA

578 amplicon and then the difference between control (only Albugo) and treatment (Albugo+

579 GH25/Mut_GH25) was calculated by ddCq. The relative biomass of Albugo was

580 analyzed by the formula $\left(2^{-\mathrm{ddCq}}\right)$. Each data point in the graph represent three

581 independent biological replicates.

582

583

\section{Nucleic acid methods}

584 RNA-Extraction of Latex-peeled samples: Four weeks old $A$. thaliana plants were fixed 585 between two fingers and liquid latex was applied to the leaf surface by using a small

586 brush. The latex was dried using the cold air option of a hair dryer, carefully peeled off with a thin tweezer and immediately frozen in liquid nitrogen. Afterwards, the frozen latex pieces were grinded with liquid nitrogen and the RNA was isolated by using Trizol $^{\circledR}$ Reagent (Invitrogen, Karlsruhe, Germany) according to the manufacturer's instructions. Turbo DNA-Free ${ }^{\mathrm{TM}}$ Kit (Ambion, life technologies ${ }^{\mathrm{TM}}$, Carlsbad, California, USA) was used to remove any DNA contamination in the extracted RNA. Synthesis of cDNA was 592 performed using First Strand cDNA Synthesis Kit (Thermo Fischer scientific, Waltham,

593 Massachusetts, USA) according to recommended instruction starting with a 594 concentration of $10 \mu \mathrm{g}$ RNA. QIAprep Mini Plasmid Prep Kit (QIAGEN, Venlo, 595 Netherlands) was used for isolation of plasmid DNA from bacteria after the principle of 596 alkaline lysis. Genomic DNA was isolated using phenol-chloroform extraction protocol 597 (18).

598 RT-qPCR oligonucleotide pairs were designed with Primer3 Plus. The oligonucleotide 599 pairs were at first tested for efficiency using a dilution series of genomic DNA. The 600 reaction was performed in a Bio-Rad iCycler system using the following conditions: 2 $601 \mathrm{~min}$ at $95^{\circ} \mathrm{C}$, followed by 45 cycles of $30 \mathrm{~s}$ at $95^{\circ} \mathrm{C}, 30 \mathrm{~s}$ at $61^{\circ} \mathrm{C}$ and generation of 602 melting curve between $65^{\circ} \mathrm{C}$ to $95^{\circ} \mathrm{C}$.

603

604 Bioinformatics and computational data analysis

605 Sequence assembly of MbA strains was performed using the HGAP pipeline (Pacific 606 Biosciences). MbA genome was annotated with the Augustus software tool. Secretome 607 was investigated using SignalP4.0. Analysis of functional domains in the secreted 
608 proteins was done by Inter-Pro Scan. AntiSmash was used to predict potential 609 secondary metabolite clusters. RNA sequencing was done at the CCG- Cologne Center 610 for Genomics by using a poly-A enrichment on an Illumina HiSeq4000 platform. The 611 achieved paired end reads were mapped to the MbA and $A$. thaliana TAIR10 genome 612 by using Tophat2 (28). RNA-Seq reads of MbA axenic cultures were used to generate 613 exon and intron hints and to start a second annotation with Augustus. Heat-maps were 614 performed using the heatmap.2 function of the package gplots (version 3.0.1) in r-studio 615 (R version 3.5.1). An analysis of variance (ANOVA) model was used for pairwise 616 comparison of the conditions, with Tukey\&apos;S HSD test to determine significant 617 differences among them $(P$ values $<0.05)$.

\section{Heterologous protein production and GH25 activity assay}

620 The Pichia pastoris $\mathrm{KM} 71 \mathrm{H}-\mathrm{OCH}$ gene expression system was used to produce MBA_GH25 domain tagged with an N-terminal Pilyhistidine tah (6XHis) and a C-

622 terminal peptide containing the c-myc epitope and a 6xHis tag. The His-MspGH25 623 cloned into pGAPZaA vector (Invitrogen, Carlsbad, CA, USA) under the control of a 624 constitutive promotor with an a-factor signal peptide for secretion. Expression and 625 purification of recombinant proteins were performed according to manufacturer's 626 instructions (Invitrogen Corporation, Catalog no. K1710-01): YPD medium 627 supplemented with $100 \mathrm{\mu g} \mathrm{ml}^{-1}$ zeocin was used for initial growth of $P$. pastoris strains at $62828^{\circ} \mathrm{C}$ and $200 \mathrm{rpm}$ (for liquid cultures). Production of the recombinant protein was 629 performed in $1 \mathrm{~L}$ buffered (100 mM Potassium phosphate buffer, pH 6.0) YPD medium 630 with $2 \%$ Sucrose at $28^{\circ} \mathrm{C}$ for 24 hours with $200 \mathrm{rpm}$ shaking. Next the protein was 631 subjected to affinity purification with a Ni-NTA-matrix, according to manufacturer's 632 instructions (Ni-Sepharose ${ }^{\mathrm{TM}} 6$ Fast-Flow, GE-Healthcare; Freiburg, Germany). After 633 purification, the His-MspGH25 protein was dialyzed in an exchange buffer $(0.1 \mathrm{M} \mathrm{NaPi}$, $6340.1 \mathrm{M} \mathrm{Nacl}, \mathrm{pH}=7.5)$. The purified protein was kept in $100 \mu \mathrm{l}$ aliquots at $4^{\circ} \mathrm{C}$.

635 Site directed mutagenesis was performed on pGAPZa-His- MspGH25 vector according 636 to the instructions of the QuikChange Multi Site-Directed Mutagenesis Kit (Agilent 637 Technologies, Santa Clara, United States) with primers targeting nucleotides of the 638 active site of $\mathrm{GH} 25$. 
639 Purified Glycoside Hydrolase of $M B A$ from P. pastoris was quantified according to a 640 sensitive fluorescence-based method using Molecular Probes ${ }^{\mathrm{TM}}$ EnzChek ${ }^{\mathrm{TM}}$ Lysozym- $^{-}$ Assay-Kit (ThermoFisher Scientific, Katalognummer: E22013). DQ lysozyme substrate

642 (Micrococcus lysodeikticus) stock suspension $(1.0 \mathrm{mg} / \mathrm{ml}$ ) and $1000 \mathrm{units} / \mathrm{ml}$ Hen egg 643 White Lysozyme (HEWL) stock solution were prepared according to the manufacturer. 644 Molar concentration of the HEWL stock solution was calculated using the following 645 website (https://www.bioline.com/media/calculator/01 04.html) and was found to be $64611 \mu \mathrm{M}$. Protein concentration of MspGH25 (both active and mutated version was 647 measured in the Nanodrop 2000c spectrophotometer (Thermo Fischer scientific, 648 Waltham, Massachusetts, USA) according to manufacturer's instructions using $100 \mu$ l of 649 sample after using $100 \mu \mathrm{l}$ of the appropriate buffer as a blank control in glass cuvette. 650 The molar concentrations of recombinant proteins were also calculated as above.

651 Starting the reaction $50 \mu$ l of the DQ lysozyme substrate working suspension was added 652 to each microplate well containing reaction buffer with either HEWL (in molar 653 concentrations ranging from $0.1-5.5 \mu \mathrm{M}$ ) or MspGH25 (in molar concentration from 0.5$65417.5 \mu \mathrm{M})$. Fluorescence intensity of each reaction was measured every $5 \mathrm{~min}$ to follow 655 the kinetic of the reaction at $37^{\circ} \mathrm{C}$ for $60 \mathrm{~min}$, using fluorescence microplate reader with 656 fluorescein filter Tecan Infinite 200 Pro plate reader (Tecan Group Ltd., Männendorf, 657 Switzerland). Digestion products from the DQ lysozyme substrate have an absorption 658 maximum at $\sim 494 \mathrm{~nm}$ and a fluorescence emission maximum at $\sim 518 \mathrm{~nm}$.

659

660

661

Data availability

662 Genome information and RNA sequencing have been submitted to NCBI Genbank and 663 are available under the following links:

664 https://www.ncbi.nlm.nih.gov/geo/query/acc.cgi?acc=GSE148670

665

666

\section{Acknowledgments}

668 This work was funded through by the Deutsche Forschungsgemeinschaft (DFG, 669 German Research Foundation) under Germany's Excellence Strategy EXC-2048/1, 
Project ID 390686111, and the DFG priority program SPP2125 "DECRyPT". We are grateful to Marco Thines for generously providing $M$. bullatus wild type strains. We thank Libera Lo Presti for critically reading the manuscript and helpful comments and suggestion.

\section{References}

1. Ritpitakphong U, Falquet L, Vimoltust A, Berger A, Métraux JP, L'Haridon F. The microbiome of the leaf surface of Arabidopsis protects against a fungal pathogen. New Phytologist. 2016;

2. Walker TS, Bais HP, Grotewold E, Vivanco JM. Root exudation and rhizosphere biology. Plant Physiology. 2003;

3. Bulgarelli D, Schlaeppi K, Spaepen S, van Themaat EVL, Schulze-Lefert P. Structure and Functions of the Bacterial Microbiota of Plants. Annual Review of Plant Biology. 2013;

4. Busby PE, Peay KG, Newcombe G. Common foliar fungi of Populus trichocarpa modify Melampsora rust disease severity. New Phytologist. 2016;

5. Mikiciński A, Sobiczewski P, Puławska J, Maciorowski R. Control of fire blight (Erwinia amylovora) by a novel strain $49 \mathrm{M}$ of Pseudomonas graminis from the phyllosphere of apple (Malus spp.). European Journal of Plant Pathology. 2016;

6. Stone BWG, Weingarten EA, Jackson CR. The Role of the Phyllosphere Microbiome in Plant Health and Function. In: Annual Plant Reviews online. 2018.

7. Agler MT, Ruhe J, Kroll S, Morhenn C, Kim ST, Weigel D, et al. Microbial Hub Taxa Link Host and Abiotic Factors to Plant Microbiome Variation. PLoS Biology. 2016;

8. Coyte KZ, Schluter J, Foster KR. The ecology of the mCoyte, K.Z., Schluter, J. \& Foster, K.R. (2015) The ecology of the microbiome: Networks, competition, and stability. Science, 350, 663-666.icrobiome: Networks, competition, and stability. Science. 2015;

9. Vorholt JA. Microbial life in the phyllosphere. Nature Reviews Microbiology. 2012;

10. Brefort T, Doehlemann G, Mendoza-Mendoza A, Reissmann S, Djamei A, Kahmann R. Ustilago maydis as a Pathogen. Annual Review of Phytopathology. 2009;

11. Kruse J, Doehlemann G, Kemen E, Thines M. Asexual and sexual morphs of Moesziomyces revisited. IMA Fungus. 2017; 
12. Wang QM, Begerow D, Groenewald M, Liu XZ, Theelen B, Bai FY, et al. Multigene phylogeny and taxonomic revision of yeasts and related fungi in the Ustilaginomycotina. Studies in Mycology. 2015;81:55-83.

13. Barda O, Shalev O, Alster S, Buxdorf K, Gafni A, Levy M. Pseudozyma aphidis induces salicylic-acid-independent resistance to Clavibacter michiganensis in tomato plants. Plant Disease. 2015;

14. Gafni A, Calderon CE, Harris R, Buxdorf K, Dafa-Berger A, Zeilinger-Reichert E, et al. Biological control of the cucurbit powdery mildew pathogen Podosphaera xanthii by means of the epiphytic fungus Pseudozyma aphidis and parasitism as a mode of action. Frontiers in Plant Science. 2015;

15. Lee G, Lee SH, Kim KM, Ryu CM. Foliar application of the leaf-colonizing yeast Pseudozyma churashimaensis elicits systemic defense of pepper against bacterial and viral pathogens. Scientific Reports. 2017;

16. Zuo W, Ökmen B, Depotter JRL, Ebert MK, Redkar A, Misas Villamil J, et al. Molecular Interactions Between Smut Fungi and Their Host Plants. Annual Review of Phytopathology. 2019;

17. Stanke M, Steinkamp R, Waack S, Morgenstern B. AUGUSTUS: A web server for gene finding in eukaryotes. Nucleic Acids Research. 2004;

18. Kämper J, Kahmann R, Bölker M, Ma LJ, Brefort T, Saville BJ, et al. Insights from the genome of the biotrophic fungal plant pathogen Ustilago maydis. Nature. 2006;

19. Redkar A, Hoser R, Schilling L, Zechmann B, Krzymowska M, Walbot V, et al. A secreted effector protein of Ustilago maydis guides maize leaf cells to form tumors. Plant Cell. 2015;

20. Schirawski J, Mannhaupt G, Münch K, Brefort T, Schipper K, Doehlemann G, et al. Pathogenicity determinants in smut fungi revealed by genome comparison. Science. 2010;

21. Bölker M, Urban M, Kahmann R. The a mating type locus of U. maydis specifies cell signaling components. Cell. 1992;

22. Lefebvre F, Joly DL, Labbé C, Teichmann B, Linning R, Belzile F, et al. The transition from a phytopathogenic smut ancestor to an anamorphic biocontrol agent deciphered by comparative whole-genome analysis. Plant Cell. 2013;

23. Sharma R, Ökmen B, Doehlemann G, Thines M. Saprotrophic yeasts formerly classified as Pseudozyma have retained a large effector arsenal, including functional Pep1 orthologs. Mycological Progress. 2019; 
24. Brefort T, Tanaka S, Neidig N, Doehlemann G, Vincon V, Kahmann R. Characterization of the Largest Effector Gene Cluster of Ustilago maydis. PLoS Pathogens. 2014;

25. Dutheil JY, Mannhaupt G, Schweizer G, Sieber CMK, Münsterkötter M, Güldener $U$, et al. A tale of genome compartmentalization: The evolution of virulence clusters in smut fungi. Genome Biology and Evolution. 2016;

26. Khrunyk Y, Münch K, Schipper K, Lupas AN, Kahmann R. The use of FLPmediated recombination for the functional analysis of an effector gene family in the biotrophic smut fungus Ustilago maydis. New Phytologist. 2010;

27. Mendoza-Mendoza A, Berndt P, Djamei A, Weise C, Linne U, Marahiel M, et al. Physical-chemical plant-derived signals induce differentiation in Ustilago maydis. Molecular Microbiology. 2009;

28. Kim D, Pertea G, Trapnell C, Pimentel H, Kelley R, Salzberg SL. TopHat2: Accurate alignment of transcriptomes in the presence of insertions, deletions and gene fusions. Genome Biology. 2013;

29. Vannier N, Agler M, Hacquard S. Microbiota-mediated disease resistance in plants. PLoS Pathogens. 2019;

30. Durán P, Thiergart T, Garrido-Oter R, Agler M, Kemen E, Schulze-Lefert P, et al. Microbial Interkingdom Interactions in Roots Promote Arabidopsis Survival. Cell. 2018;

31. Helfrich EJN, Vogel CM, Ueoka R, Schäfer M, Ryffel F, Müller DB, et al. Bipartite interactions, antibiotic production and biosynthetic potential of the Arabidopsis leaf microbiome. Nature Microbiology. 2018;

32. Boekhout T. Pseudozyma Bandoni emend: Boekhout (1985) and a comparison with the yeast state of Ustilago maydis (De Candolle) Corda (1842). In: The Yeasts. 2011.

33. Kitamoto D, Akiba S, Hioki C, Tabuchi T. Extracellular accumulation of mannosylerythritol lipids by a strain of candida antarctica. Agricultural and Biological Chemistry. 1990;

34. Morita T, Konishi M, Fukuoka T, Imura T, Kitamoto D. Microbial conversion of glycerol into glycolipid biosurfactants, mannosylerythritol lipids, by a basidiomycete yeast, Pseudozyma antarctica JCM 10317T. Journal of Bioscience and Bioengineering. 2007;

35. Cheng Y, McNally DJ, Labbé C, Voyer N, Belzile F, Bélanger RR. Insertional mutagenesis of a fungal biocontrol agent led to discovery of a rare cellobiose lipid with antifungal activity. Applied and Environmental Microbiology. 2003; 
36. Mimee B, Labbé C, Pelletier R, Bélanger RR. Antifungal activity of flocculosin, a novel glycolipid isolated from Pseudozyma flocculosa. Antimicrobial Agents and Chemotherapy. 2005;

37. Teichmann B, Linne U, Hewald S, Marahiel MA, Bölker M. A biosynthetic gene cluster for a secreted cellobiose lipid with antifungal activity from Ustilago maydis. Molecular Microbiology. 2007;

38. Schroeckh V, Scherlach K, Nützmann HW, Shelest E, Schmidt-Heck W, Schuemann J, et al. Intimate bacterial-fungal interaction triggers biosynthesis of archetypal polyketides in Aspergillus nidulans. Proceedings of the National Academy of Sciences of the United States of America. 2009;

39. Rutledge PJ, Challis GL. Discovery of microbial natural products by activation of silent biosynthetic gene clusters. Nature Reviews Microbiology. 2015;

40. Stroe MC, Netzker T, Scherlach K, Krüger T, Hertweck C, Valiante V, et al. Targeted induction of a silent fungal gene cluster encoding the bacteria-specific germination inhibitor fumigermin. eLife. 2020;

41. Bölker M. Ustilago maydis - A valuable model system for the study of fungal dimorphism and virulence. Microbiology. 2001;

42. Nadal M, Gold SE. The autophagy genes atg8 and atg1 affect morphogenesis and pathogenicity in Ustilago maydis. Molecular Plant Pathology. 2010;

43. Rabe F, Bosch J, Stirnberg A, Guse T, Bauer L, Seitner D, et al. A complete toolset for the study of Ustilago bromivora and Brachypodium sp. as a fungal-temperate grass pathosystem. eLife. 2016;

44. Chen Y, Miyata S, Makino S, Moriyama R. Molecular characterization of a germination-specific muramidase from Clostridium perfringens $\mathrm{S} 40$ spores and nucleotide sequence of the corresponding gene. Journal of Bacteriology. 1997;

45. Rau A, Hogg T, Marquardt R, Hilgenfeld R. A new lysozyme fold. Crystal structure of the muramidase from Streptomyces coelicolor at $1.65 \AA$ resolution. Journal of Biological Chemistry. 2001;

46. Korczynska JE, Danielsen S, Schagerlöf U, Turkenburg JP, Davies GJ, Wilson KS, et al. The structure of a family GH25 lysozyme from Aspergillus fumigatus. Acta Crystallographica Section F: Structural Biology and Crystallization Communications. 2010;

47. Hyde KD, Xu J, Rapior S, Jeewon R, Lumyong S, Niego AGT, et al. The amazing potential of fungi: 50 ways we can exploit fungi industrially. Fungal Diversity. 2019; 
48. Horner NR, Grenville-Briggs LJ, van West P. The oomycete Pythium oligandrum expresses putative effectors during mycoparasitism of Phytophthora infestans and is amenable to transformation. Fungal Biology. 2012;

49. Parratt SR, Laine AL. The role of hyperparasitism in microbial pathogen ecology and evolution. ISME Journal. 2016;

50. Kämper J. A PCR-based system for highly efficient generation of gene replacement mutants in Ustilago maydis. Molecular Genetics and Genomics. 2004;

51. Ruhe J, Agler MT, Placzek A, Kramer K, Finkemeier I, Kemen EM. Obligate biotroph pathogens of the genus albugo are better adapted to active host defense compared to niche competitors. Frontiers in Plant Science. 2016;

52. Hemetsberger C, Mueller AN, Matei A, Herrberger C, Hensel G, Kumlehn J, et al. The fungal core effector Pep1 is conserved across smuts of dicots and monocots. New Phytologist. 2015;206(3):1116-1126.

53. Doehlemann G, Van Der Linde K, Aßmann D, Schwammbach D, Hof A, Mohanty A, et al. Pep1, a secreted effector protein of Ustilago maydis, is required for successful invasion of plant cells. PLoS Pathogens. 2009;

54. Schilling L, Matei A, Redkar A, Walbot V, Doehlemann G. Virulence of the maize smut Ustilago maydis is shaped by organ-specific effectors. Molecular Plant Pathology. 2014;

55. Seitner D, Uhse S, Gallei M, Djamei A. The core effector Cce1 is required for early infection of maize by Ustilago maydis. Molecular Plant Pathology. 2018;

56. Krombach S, Reissmann S, Kreibich S, Bochen F, Kahmann R. Virulence function of the Ustilago maydis sterol carrier protein 2. New Phytologist. 2018;

57. Schipper K. Charakterisierung eines Ustilago maydis Genclusters, das f€ur drei neuartige sekretierte Effektoren kodiert. PhDitle. PhD thesis. 2009;

58. Doehlemann G, Reissmann S, Aßmann D, Fleckenstein M, Kahmann R. Two linked genes encoding a secreted effector and a membrane protein are essential for Ustilago maydis-induced tumour formation. Molecular Microbiology. 2011;

59. Ma LS, Wang L, Trippel C, Mendoza-Mendoza A, Ullmann S, Moretti M, et al. The Ustilago maydis repetitive effector Rsp3 blocks the antifungal activity of mannosebinding maize proteins. Nature Communications. 2018;

60. Djamei A, Schipper K, Rabe F, Ghosh A, Vincon V, Kahnt J, et al. Metabolic priming by a secreted fungal effector. Nature. 2011; 
bioRxiv preprint doi: https://doi.org/10.1101/2020 .04 20.051367 this version posted December 2, 2020. The copyright holder for this preprint (which was not certified by peer review) is the author/funder, who has granted bioRxiv a license to display the preprint in perpetuity. It is made available under aCC-BY-NC-ND 4.0 International license.

841 61. Ökmen B, Kemmerich B, Hilbig D, Wemhöner R, Aschenbroich J, Perrar A, et al. 842 Dual function of a secreted fungalysin metalloprotease in Ustilago maydis. New $843 \quad$ Phytologist. 2018;

844

845

846

847

848 
849

850

851

852

853

854

855

856

857

858

859

860

861

862

863

864

865

866

867

868

869

870

871

872

873

874

875

876

877

878

\section{Supporting information captions}

Figure S1: Biocontrol activity of $M b A$, but not $U$. maydis, against bacterial SynCom members (7). Inhibition by Moesziomyces can be seen as a characteristic halo after 48hrs of co-incubation.

Figure S2: Trypan blue staining of $A$. thaliana leaves 15 days post infection with $A$. laibachii. (i) \& (ii)- control set with only A.laibachii (error bar $50 \mu \mathrm{m}$ ) ; (i)- Thick hyphal growth of A.laibachii on the leaf surface, (ii) appressoria formation can be seen (red arrow). (iii) \& (iv) Treatment set (MbA sprayed two days before Albugo (iii): Zoospores aggregated together, with few of them forming hyphae (green box); in addition, short, broken hyphae visualized in some regions (red box), which have not been found in

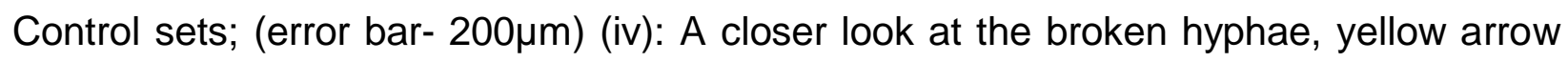

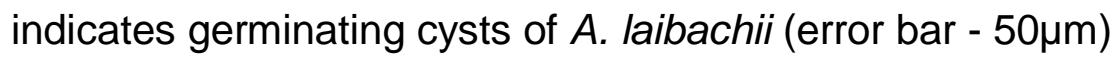

Figure S3: Genome comparison of $M b A$ and Moesziomyces antarctica T-34. Highlighted regions show that contigs with chromosomal rearrangements in MBA can be also found in the genome of the related species Moesziomyces antarctica T-34.

Figure S4: (A) Predicted secondary metabolite clusters in the genome of $M b A$. Most clusters have unpredictable functions, three belong to the type of terpene or nonribosomal peptide synthetase types and one is a polyketide synthetase cluster type I. (B) The gene cluster encoding for production of ustilagic acid,a well-studied secondary metabolite of smut fungi (37), is not present in the genome of $M b A$. (C) Out of the 13 predicted secondary metabolite clusters, three are unique to $M b A$. Cluster 2 is predicted to encode a terpene, cluster 8 is a cluster of unknown function and cluster 10 is predicted as NRPS cluster. Core biosynthetic genes are highlighted in red, additional biosynthetic genes in yellow and transport-related genes in blue, based on AntiSMASH predictions. 
879

880

881

882

883

884

885

886

887

888

889

890

891

892

893

894

895

896

897

898

899

900

901

902

903

904

905

906

907

908

909

Figure S5: Protein alignment of the core effector Pep1 (52) from different Ustilaginomycetes. (A) Pep1 regions important for functionality are present in all the aligned sequences. Deletion of the pep1 gene (UMAG_01987) in U. maydis leads to complete loss of virulence, which can be restored by complementing the deletion mutant with the MbA pep1 gene (Ma1682). Infection of maize leaves was done as described in (19). Disease symptoms were scored at 12 days post infection in three independent biological replicates. $n=$ number of plants infected

Figure S6: Comparison of known virulence clusters (18) between U. maydis and $M b A$. Numbers are gene numbers (*UMAG_NUMBER ${ }^{*}$ for U. maydis; ${ }^{*}$ gNUMBER* for $M b A$ ).

Figure S7: Mating assays of $M b A$ and different $M$. bullatus isolates. Mixing of haploid $U$. maydis strains FB1 \& FB2 and the solopathogenic strain SG200 served as a positive control for mating on charcoal plates, wherein filamentous growth is indicated by white, fluffy appearance of colonies. Haploid wild type strains without mating partner don't show a fluffy phenotype. For all combinations of Moesziomyces strains, no mating event resulting in filamentous growth on charcoal plates was observed.

Figure S8: A) Multi-dimensional scaling plot (MDS) plot based on the interactions of Moesziomyces sp. (M.sp.) / MbA in response to the SynCom bacteria and Albugo laibachii in three biological replicates. The MDS plot shows Albugo and nonAlbugo samples grouping together based on gene-level logCPM. (B) Voom meanvariance trend of the dataset where points represent genes, and $(C)$ sample-specific weights obtained from the limma-voom function. Colours represent three replicates for each treatment. Light blue: $M b A$ on plant; Dark blue: $M b A$ on plant + SynCom; Light green: $M b A$ on plant + Albugo; Dark green: MbA on plant + Albugo + SynCom .

Figure S9: (A) Sequence distribution of Gene Ontology terms. $60 \%$ of all the genes that are downregulated in $M b A$ on plant compared to axenic culture growth can be assigned to GO-terms related to metabolism and cell cycle. (B) In contrast, presence of $A$. laibachii leads to transcriptional activation of metabolic processes. $52 \%$ of all GO-terms 
910

911

912

913

914

915

916

917

918

919

920

921

922

923

924

925

926

927

928

929

930

931

932

933

934

935

936

937

938

939

940

associated with genes upregulated in presence of $A$. laibachii are related to metabolic processes.

Figure S10: Stress assay of $M b A$ wild type and knockout mutants of gene (g5, g5755 and g2490) respectively, on CM medium and $2 \%$ Glucose (A) with different conditions (B: $100 \mu \mathrm{g} / \mathrm{ml}$ Calcofluor; C: $150 \mu \mathrm{g} / \mathrm{ml}$ Calcoflour; D: $1 \mathrm{mMH} 2 \mathrm{O} 2 ; \mathrm{E}: 45$ $\mu \mathrm{g} / \mathrm{ml}$ Congored; F: $1 \mathrm{M} \mathrm{NaCl}$; G: $1 \mathrm{M}$ Sorbitol). The strains were dropped on the $\mathrm{CM}$ plates containing different stress supplements in a dilution series from $10^{0}$ to $10^{-4}$

Figure S11: A) Recombinant MbA_GH25 was produced and purified using the Pichia pastoris protein expression system. The purified protein was loaded in a $12 \%$ SDS gel for visualization of an expected molecular weight of $27 \mathrm{kDa}$ for His-Tagged GH25. B) Schematic diagram of the recombinant construct, where the GH25 domain from MBA is tagged with an $\mathrm{N}$-terminal polyhistidine Tag and a C-terminal peptide containing the cmyc epitope and a polyhistidine tag. C) Detection of lysozyme activity for Commercial Hen-egg white lysozyme (stock solution, $11 \mu \mathrm{M}$ ) using the EnzChek ${ }^{1}$ Lysozyme Assay Kit. The fluorescence was recorded every minute in a fluorescence microplate reader using excitation/emission of $485 / 530 \mathrm{~nm}$ in increasing concentrations from $0.1 \mu \mathrm{M}$ to 5.5 $\mu \mathrm{M}$. Finally, Relative Fluorescence Unit (RFU)/ $\min$ was calculated for each concentration and plotted on the graph.

Figure S12: A molecular phylogenetic analysis using maximum likelihood estimation and based on pheromone receptor protein sequences similarity. MbA protein sequence clusters together with type 1 pheromone receptors of other Ustilaginomycetes.

Figure S13: Amino acid alignment of GH25 sequences from different fungi (see attached list -'GH25 with accession number', for full length sequences). The protein sequences were obtained from the NCBI database. Alignment was achieved using the PRALINE multiple sequence alignment program with default parameters. The scoring scheme works from 0 for the least conserved alignment position, up to 10 (indicated by *) for the most conserved alignment position. A conserved active-site DxE motif has 
941 been predicted for glycoside hydrolase family 25. Sequences tested from different

942 basiodiomycete, ascomycete and Chytrids, have the active site residue conserved 943 (purple box).

944

945 Figure S14: Boxplot-analysis of $\mathrm{GH} 25$ treatment on in vitro A. laibachii zoosporangial germination in three biological replicates analyzing about 100 zoosporangial cells for each replicate. A $\mathrm{p}$ value of 0.3 was obtained for paired T-test using one-tailed

948 distribution.

949

Table S1: Composition of the bacterial SynCom

Table S2: MbA gene expression data

952 Table S3: Growth media and buffers used in this study

953 Table S4: PCR primers used in this study

954

955

\section{Figure Legends}

956

Figure 1: Position of $M b A$ in the family of Ustilaginaceae. (A) molecular phylogenetic 958 analysis by maximum likelihood method based on fungal ITS sequences and showing grouping of $M b A$ with the millet pathogen $M$. bullatus. Pathogenic filamentous smuts (green) and anamorphic smut yeasts can be found across the phylogenetic tree. (B) Comparison of the morphology of $M b A$ (bottom) and $U$. maydis (top) haploid cells grown in $\mathrm{YEPSl}_{\text {ight }}$ medium to $\mathrm{OD}_{600} 0.6$.

Figure 2: Infection assay of $A$. laibachii on $A$. thaliana. Addition of a bacterial SynCom reduces the infection symptoms of $A$. laibachii at $14 \mathrm{dpi}$. Those symptoms can be almost abolished by spraying $M b A$ to the plant, independently of the presence of the bacterial

967 community. Infections were performed in six individual replicates with 12 technical 968 replicates. $\mathrm{N}$ indicates the number of infected plants that were scored for symptoms. An

969 analysis of variance (ANOVA) model was used for pairwise comparison of the 970 conditions, with Tukey\&apos;s HSD test to determine differences among them. Different 971 letters indicate significant differences $(P$ values $<0.05)$. 
973 Figure 3: Circos comparison of $M b A$ and $U$. maydis chromosome structure $(\mathrm{A})$. We 974 highlighted potential secondary metabolite clusters, secreted proteins and gene 975 predictions on both strands (+/-). (B) Homology based comparisons identified three 976 chromosomal recombination events, which affects the MbA contigs 2, $6 \& 8$.

977

Figure 4: Structure of the largest virulence cluster (Cluster 19A) in pathogenic smut fungi and anamorphic smut yeasts (marked with*). Colors indicate genes with homology to each other: Related gene families are indicated in orange, yellow, blue, green and brown, whereas unique effector genes are shown in grey. Genes encoding proteins without a secretion signal are shown in white (24).

Figure 5: Genetic transformation of $M b A$. (A) Stable transformants that express cytosolic GFP could be obtained by generating protoplasts with Glucanex and 986 ectopically integrating linear DNA-fragments into the genome via PEG-mediated transformation. (B) Overview of the split-marker approach that was used to generate deletion mutants via homologous recombination.

Figure 6: The self-compatible $M b A$ strain CB1 (A) MbA mating type genes, unlike the ones of $U$. hordei, can be found on two different chromosomes similar to the tetrapolar mating type system of $U$. maydis. (B) To generate a self-compatible strain (CB1), the bmating genes of $U$.hordei were integrated at the native $M b A b$-locus. (C) Unlike the $M b A$ wild type strain (top left), strain CB1 (bottom left) shows a fluffy phenotype on charcoal 995 plates and filamentous growth. U. maydis haploid F1 strain (top right) and self996 compatible SG200 strain (bottom right) were used as negative and positive control, 997 respectively. (D,E) Induction of filamentation and appressoria formation in strain CB1 998 was studied in three independent experiments. For this around 1000 cells for filament 999 formation and around 600 cells for appressoria formation were analyzed and error bars 1000 indicate standard error. After incubation on a hydrophobic surface, both, filament and 1001 appressoria formation in strain CB1, were significantly different ( ${ }^{*}$ Chi-Square Test for 1002 Independence $-a=0,0001$ ) when compared to $M b A$ wild type and similar to the level of 
1003 the self-compatible U. maydis strain SG00. U. maydis haploid F2 strain was used as

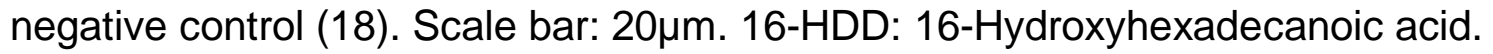

Figure 7: Transcriptome analysis of $M b A(\mathrm{~A})$ Experimental setup used for the transcriptomic (RNA-Sequencing) analysis in MbA. (B) Venn diagrams showing differential regulated $M b A$ genes after spraying of haploid cells onto the $A$. thaliana leaf surface. A total number of 801 genes were upregulated in response to $A$. laibachii in presence and absence of bacterial SynCom. 216 of the 801 genes were upregulated in both conditions. (C) Hierarchical clustering of the $27 \mathrm{~A}$. laibachii - induced MbA genes that are predicted to encode secreted proteins. Of these genes, nine were selected as candidate microbe-microbe effector genes, based on their transcriptional upregulation and prediction to encode for extracellularly localized proteins.

Figure 8: A reverse-genetic approach to identify the $M b A$ gene which is responsible for the suppression of $A$. laibachii infection. (A) Three candidate microbe-microbe effector genes ( $g 5, g 5755$ \&g2490) were deleted in MbA and deletion strains were individually inoculated on $A$. thaliana together with $A$. laibachii. Inoculation of two independent g2490 null strains ( $\Delta$ g2490_1; $\left.\Delta g 2490 \_2\right)$ resulted in significant and almost complete loss the biocontrol activity of $M b A$. While deletion of g5 resulted in a marginal reduction of disease symptoms at 14 days post infection, deletion of g5755 had no effect on A. laibachii. (B) Genetic complementation of the g2490 deletion restores the biocontrol activity to wild type levels. Infections in (A) were performed in six, in (B) in three individual replicates. In each replicate 12 plants were infected. $\mathrm{N}$ indicates the number of infected plants that were scored for symptoms. Different letters indicate significant differences ( $P$ values < 0.05; ANOVA model for pairwise comparison with Tukey\&apos;s HSD test). (C) Detection of lysozyme. Increasing concentrations of purified MbA_GH25 and MbA_GH25(D124E) were incubated with the DQ lysozyme substrate for an hour at $37^{\circ} \mathrm{C}$. The fluorescence was recorded every minute in a fluorescence microplate reader using excitation/emission of 485/530 nm. Finally, Relative Fluorescence Unit (RFU)/ min 1032 was calculated for each concentration and plotted on the graph. Each data point represents three technical replicates and three independent biological replicates as 
1034 indicated by the Standard Error Measurement (SEM) bars. An unpaired t-test was 1035 performed for the active GH25 and Mutant_GH25 sets giving the p-value of $<0.0001$; 1036 and $\mathrm{R}$ squared value of $77.24 \%$. (D) Relative quantification of $A$. laibachii biomass in 1037 response to MbA_GH25 (active and mutant) treatment via qPCR. The Oomycete 1038 internal transcribed spacer (ITS) 5.8s, was normalized to $A$. thaliana EF1-a gene to 1039 quantify the amount of $A$. laibachii DNA in the samples, ten days post infection. Then 1040 relative biomass was calculated comparing control sets (Only Albugo) with A. laibachii 1041 treated with GH25 and A. laibachii treated with Mutant_GH25 by ddCT method. 1042 Unpaired t-test between GH25 and Mutant_GH25 sets gave a p-value of $<0.0001$ and 1043 an R-squared value of $98.88 \%$.

1047 Table 1: Comparison of Genomes and genomic features of known pathogenic and 1048 anamorphic Ustilaginomycetes.

\begin{tabular}{|c|c|c|c|c|c|c|c|c|}
\hline & $M b A$ & U. bromivora & $\begin{array}{c}\text { S. } \\
\text { scitamineum }\end{array}$ & S. reilianum & U. maydis & U. hordei & $\begin{array}{c}M . \\
\text { pennsylvanicum }\end{array}$ & A. flocculosa \\
\hline \multicolumn{9}{|l|}{$\begin{array}{l}\text { Assembly } \\
\text { statistics }\end{array}$} \\
\hline $\begin{array}{l}\text { Total contig } \\
\text { length }(\mathrm{Mb})\end{array}$ & 18.3 & & 19.5 & 18.2 & 19.7 & 20.7 & 19.2 & 23.2 \\
\hline $\begin{array}{l}\text { Total scaffold } \\
\text { length }(\mathrm{Mb})\end{array}$ & & 20.5 & 19.6 & 18.4 & 19.8 & 21.15 & 19.2 & 23.3 \\
\hline $\begin{array}{l}\text { Average base } \\
\text { coverage }\end{array}$ & $50 x$ & $154 x$ & $30 x$ & $20 x$ & $10 x$ & $25 x$ & $339 x$ & $28 x$ \\
\hline $\begin{array}{l}\text { N50 contig } \\
\text { (kb) }\end{array}$ & 705.1 & & 37.6 & 50.3 & 127.4 & 48.7 & 43.4 & 38.6 \\
\hline $\begin{array}{l}\text { N50 scaffold } \\
\text { length }(\mathrm{kb})\end{array}$ & & 877 & 759.2 & 738.5 & 817.8 & 307.7 & 121.7 & 919.9 \\
\hline Chromosomes & 21 & 23 & & 23 & 23 & 23 & & \\
\hline $\begin{array}{c}\text { GC-content } \\
(\%)\end{array}$ & 60.9 & 52.4 & 54.4 & 59.7 & 54 & 52 & 50.9 & 65.1 \\
\hline Coding (\%) & 62.8 & 54.4 & 57.8 & 62.6 & 56.3 & 54.3 & 54 & 66.3 \\
\hline \multicolumn{9}{|l|}{ 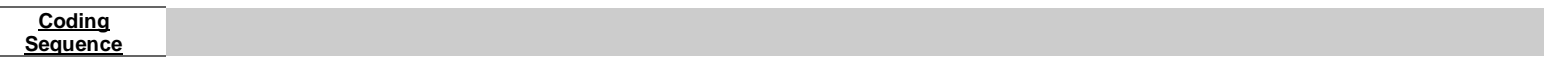 } \\
\hline $\begin{array}{l}\text { Percentage } \\
\text { CDS (\%) }\end{array}$ & 69.5 & 59.8 & 62 & 65.9 & 61.1 & 57.5 & 56.6 & 54.3 \\
\hline $\begin{array}{l}\text { Average gene } \\
\text { size (bp) }\end{array}$ & 1935 & 1699 & 1819 & 1858 & 1836 & 1708 & 1734 & 2097 \\
\hline $\begin{array}{c}\text { Average gene } \\
\text { density } \\
\text { (gene/kb) }\end{array}$ & 0.36 & 0.35 & 0.34 & 0.37 & 0.34 & 0.34 & 0.33 & 0.30 \\
\hline $\begin{array}{c}\text { Protein- } \\
\text { coding genes }\end{array}$ & 6653 & 7233 & 6693 & 6648 & 6786 & 7113 & 6279 & 6877 \\
\hline Exons & 11645 & 11154 & 10214 & 9776 & 9783 & 10907 & 9278 & 19318 \\
\hline $\begin{array}{l}\text { Average exon } \\
\text { size (bp) }\end{array}$ & 1091 & 1101 & 1191 & 1221 & 1230 & 1107 & 527 & 658 \\
\hline Exons/gene & 1.75 & 1.5 & 1.5 & 1.47 & 1.44 & 1.53 & 1.48 & 2.8 \\
\hline tRNA genes & 150 & 133 & 116 & 96 & 111 & 110 & 126 & 176 \\
\hline \multicolumn{9}{|l|}{ Noncoding } \\
\hline Introns & 9333 & 3921 & 3521 & 3103 & 2997 & 3161 & 2999 & 12427 \\
\hline Introns/gene & 1.40 & 0.54 & 0.53 & 0.47 & 0.44 & 0.44 & 0.48 & 1.81 \\
\hline $\begin{array}{c}\text { Average } \\
\text { intron length }\end{array}$ & 163 & 163 & 130.1 & 144 & 142 & 141 & 191.4 & 141 \\
\hline
\end{tabular}


bioRxiv preprint doi: https://doi.org/10.1101/2020.04.20.051367; this version posted December 2, 2020. The copyright holder for this preprint (which was not certified by peer review) is the author/funder, who has granted bioRxiv a license to display the preprint in perpetuity. It is made available under aCC-BY-NC-ND 4.0 International license.

\begin{tabular}{|c|c|c|c|c|c|c|c|c|}
\hline $\begin{array}{c}\text { (base) } \\
\text { Average } \\
\text { intergenic } \\
\text { distance (bp) }\end{array}$ & 769 & 1054 & 1114 & 929 & 1127 & 1186 & 1328 & 1273 \\
\hline \multicolumn{9}{|l|}{ Secretome } \\
\hline $\begin{array}{c}\text { Protein with } \\
\text { signal peptide }\end{array}$ & 559 & & 622 & 632 & 625 & 538 & 419 & 622 \\
\hline $\begin{array}{c}\text { Secreted } \\
\text { without TMD }\end{array}$ & 380 & & & & 467 & & & 737 \\
\hline $\begin{array}{c}\text { - with known } \\
\text { domain }\end{array}$ & 260 & & & & 264 & & & 554 \\
\hline
\end{tabular}

domain

$[24,42]$ 1051 function.

\section{Table 2: $M b A$ proteins homologous to $U$. maydis effector genes with known virulence}

\begin{tabular}{|c|c|c|c|c|c|c|}
\hline Name & Homologue & Query cover & E-value & Identity (\%) & U. maydis knockout phenotype & Reference \\
\hline g1653 & UMAG_01987 (Pep1) & $82 \%$ & $3-e 56$ & 60.96 & $\begin{array}{l}\text { complete loss of tumor formation - } \\
\text { blocked in early stages of infection }\end{array}$ & (53) \\
\hline g1828 & UMAG_01829 (Afu1) & $99 \%$ & 0.0 & 71.57 & $\begin{array}{l}\text { organ specific effector - reduced } \\
\text { virulence in seedling leaves }\end{array}$ & (54) \\
\hline g2626 & UMAG_12197 (Cce1) & $98 \%$ & $1 e-48$ & 60.16 & $\begin{array}{l}\text { complete loss of tumor formation - } \\
\text { blocked in early stages of infection }\end{array}$ & (55) \\
\hline g2765 & UMAG_11938 (Scp2) & $100 \%$ & $1 e-73$ & 93.44 & Reduced in virulence & (56) \\
\hline g2910 & UMAG_02475 (Stp1) & $32 \%$ & $3 e-42$ & 60.71 & $\begin{array}{l}\text { complete loss of tumor formation - } \\
\text { blocked in early stages of infection }\end{array}$ & (57) \\
\hline g3652 & UMAG_02239 (See1) & $43 \%$ & $9 e-11$ & 54.90 & $\begin{array}{l}\text { organ specific effector - reduced } \\
\text { virulence in seedling leaves }\end{array}$ & (19) \\
\hline g3113 & UMAG_01375 (Pit2) & * & * & * & $\begin{array}{l}\text { complete loss of tumor formation - } \\
\text { blocked in early stages of infection }\end{array}$ & (58) \\
\hline g3279 & UMAG_03274 (Rsp3) & $10 \%$ & $5 e-20$ & 70.11 & $\begin{array}{l}\text { strong attenuation of virulence - } \\
\text { reduced tumor size and number }\end{array}$ & (59) \\
\hline g5296 & UMAG_05731 (Cmu1) & $98 \%$ & $3 e-70$ & 43.84 & Reducedvirulence & $(60)$ \\
\hline g6183 & UMAG_06098 (Fly1) & $100 \%$ & 0.0 & 81.85 & Reducedvirulence & (61) \\
\hline g5835 & UMAG_05302 (Tin2) & $87 \%$ & $8 e-24$ & 37.81 & $\begin{array}{l}\text { Minor impact on tumor formation - } \\
\text { reduced anthocyanin biosynthesis }\end{array}$ & (24) \\
\hline
\end{tabular}




\section{Figure 1}

A

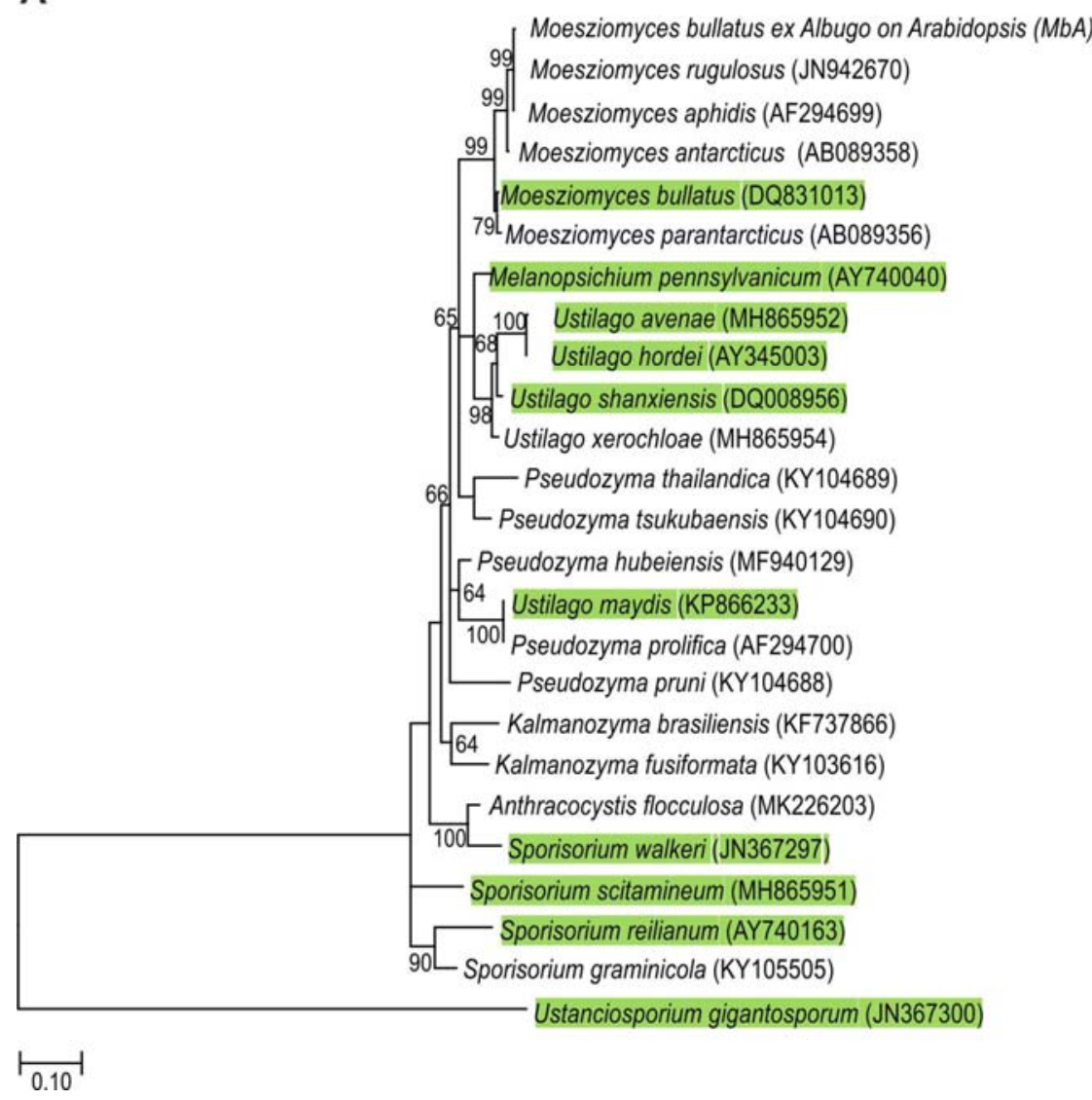

B
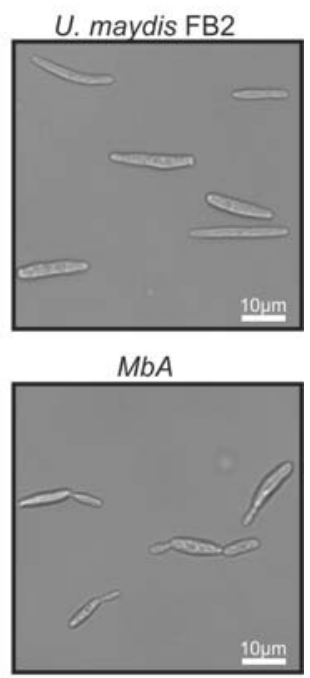

Figure 1: Position of $M b A$ in the family of Ustilaginaceae. (A) molecular phylogenetic analysis by maximum likelihood method based on fungal ITS sequences and showing grouping of $M b A$ with the millet pathogen $M$. bullatus. Pathogenic filamentous smuts (green) and anamorphic smut yeasts can be found across the phylogenetic tree. (B) Comparison of the morphology of $M b A$ (bottom) and $U$. maydis (top) haploid cells grown in YEPSlight medium to $\mathrm{OD}_{6000} 0.6$. 
Figure 2

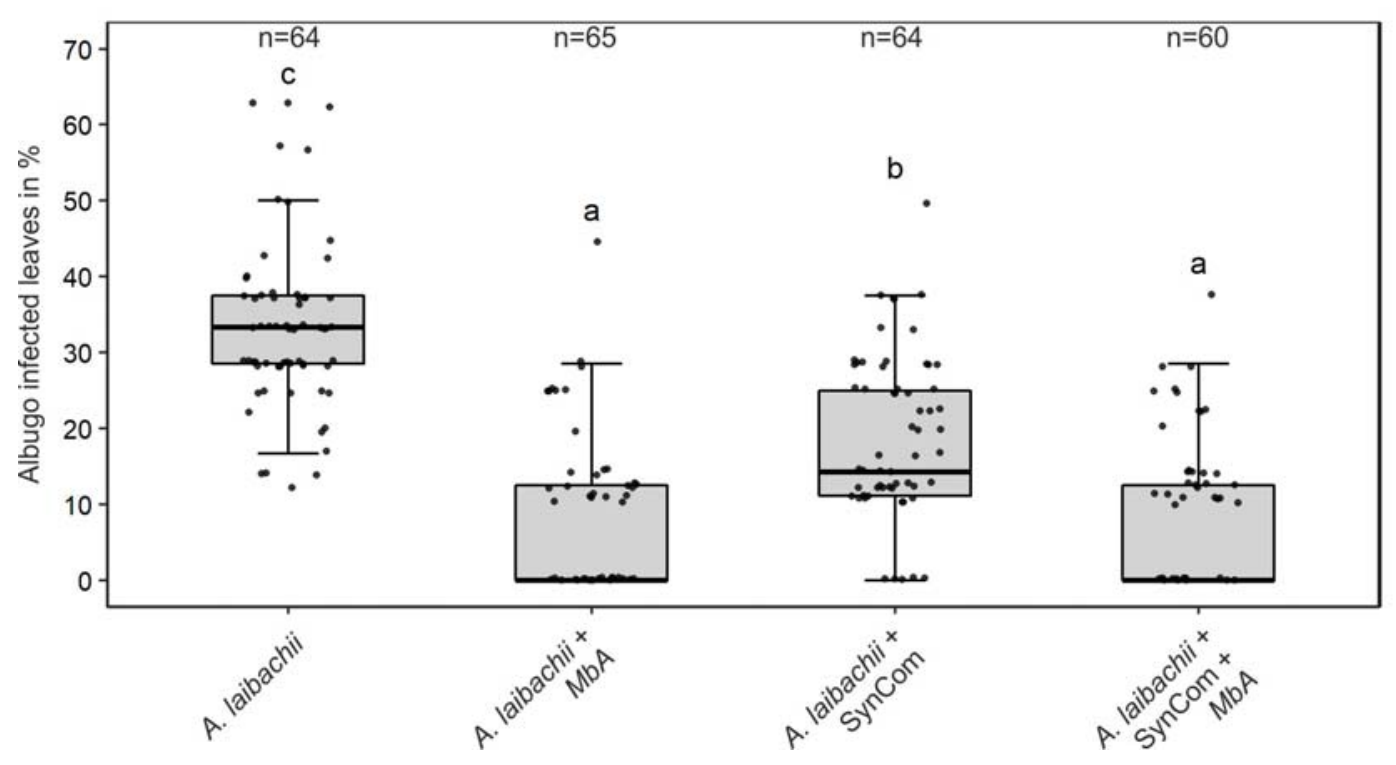

Figure 2: Infection assay of $A$. laibachii on $A$. thaliana. Addition of a bacterial SynCom reduces the infection symptoms of $A$. laibachii at $14 \mathrm{dpi}$. Those symptoms can be almost abolished by spraying $M b A$ to the plant, independently of the presence of the bacterial community. Infections were performed in six individual replicates with 12 technical replicates. $\mathrm{N}$ indicates the number of infected plants that were scored for symptoms. An analysis of variance (ANOVA) model was used for pairwise comparison of the conditions, with Tukey\&apos;s HSD test to determine differences among them. Different letters indicate significant differences $(P$ values $<0.05)$. 


\section{Figure 3}

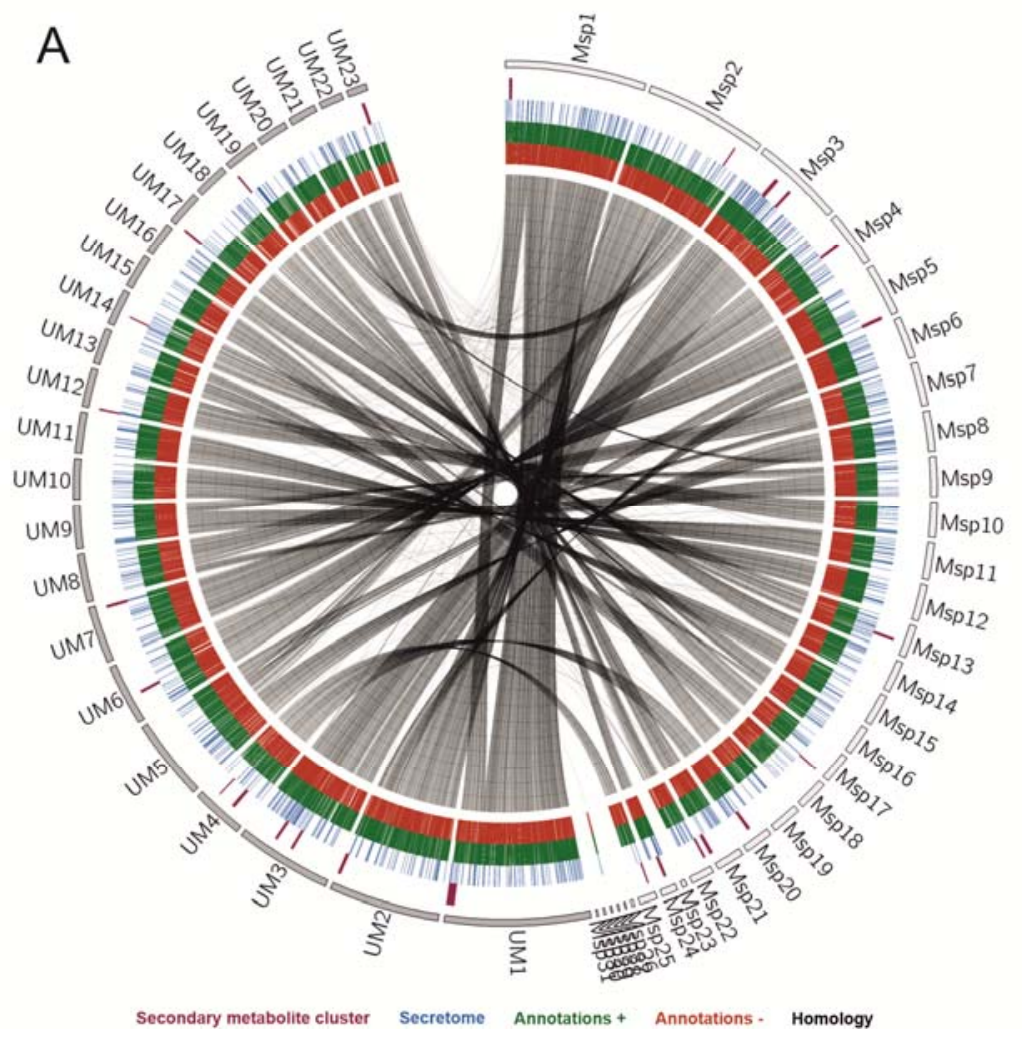

B
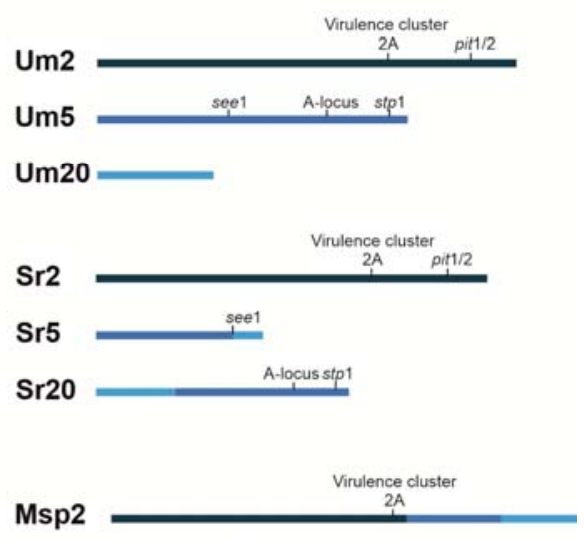

Msp6 see1

Msp8 stp A-locus piti12

see1: leat specific effector

pit 1/2 \& stp 1 : essential virulence factors

A-locus: Mating type locus A

Virulence cluster $2 A$ : associated with attenuated virulence

Secondary metabolite cluster Secretome Annotations + Annotations - Homology

Figure 3: Circos comparison of $M b A$ and $U$. maydis chromosome structure $(A)$. We highlighted potential secondary metabolite clusters, secreted proteins and gene predictions on both strands (+/-). (B) Homology based comparisons identified three chromosomal recombination events, which affects the MbA contigs 2, 6 \& 8 . 


\section{Figure 4}

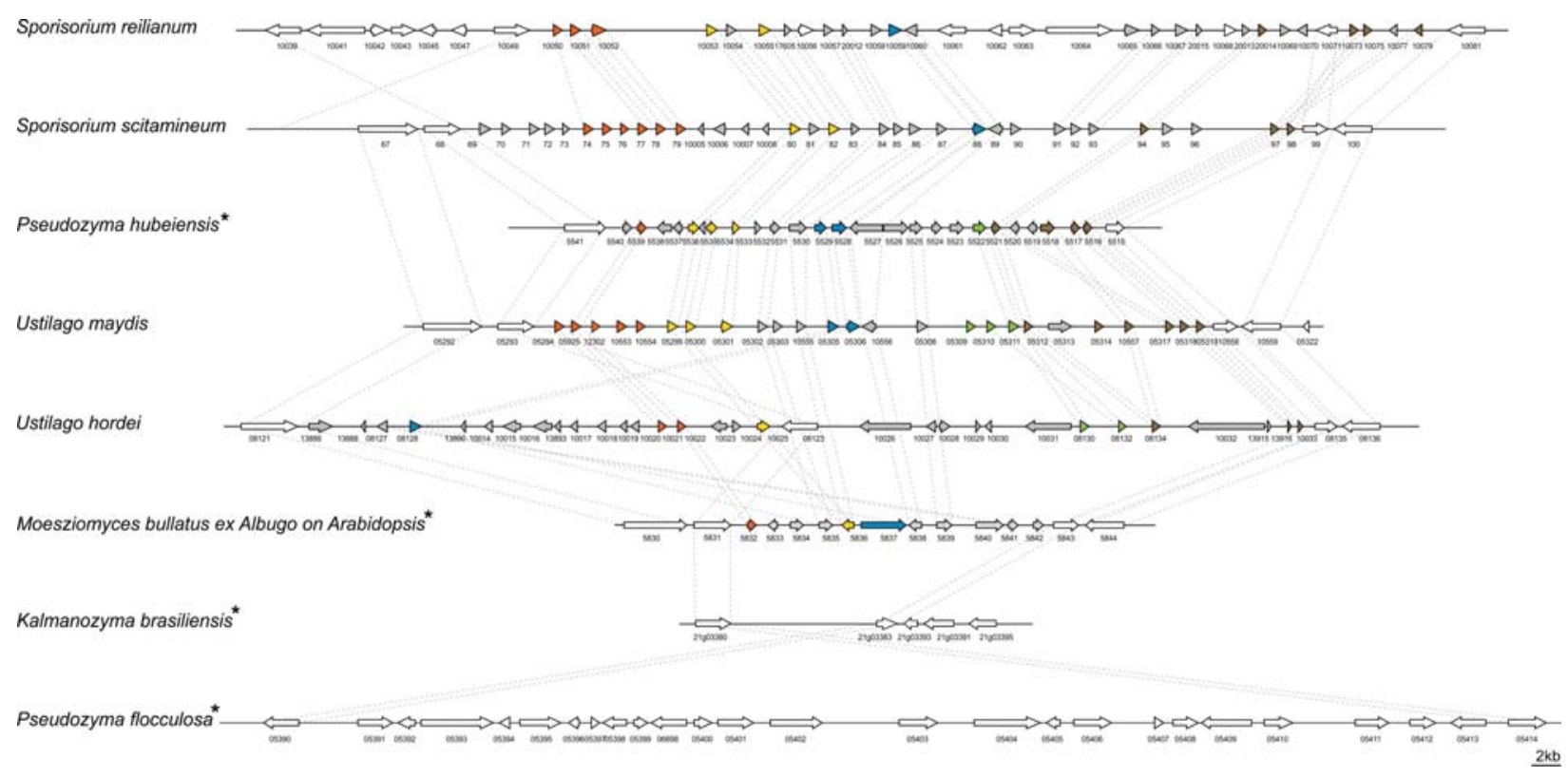

Figure 4: Structure of the largest virulence cluster (Cluster 19A) in pathogenic smut fungi and anamorphic smut yeasts (marked with*). Colors indicate genes with homology to each other: Related gene families are indicated in orange, yellow, blue, green and brown, whereas unique effector genes are shown in grey. Genes encoding proteins without a secretion signal are shown in white (24). 


\section{Figure 5}

A

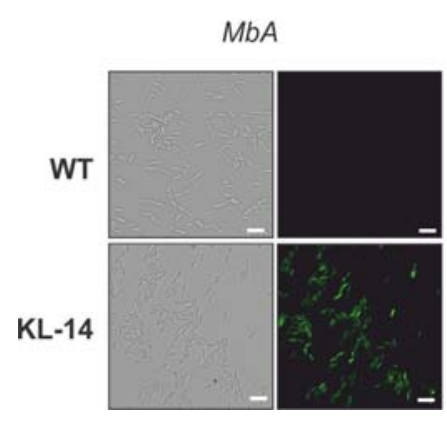

B

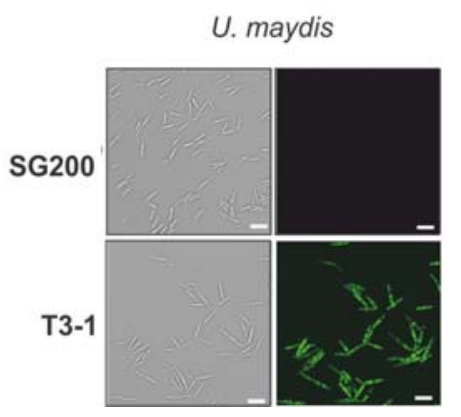

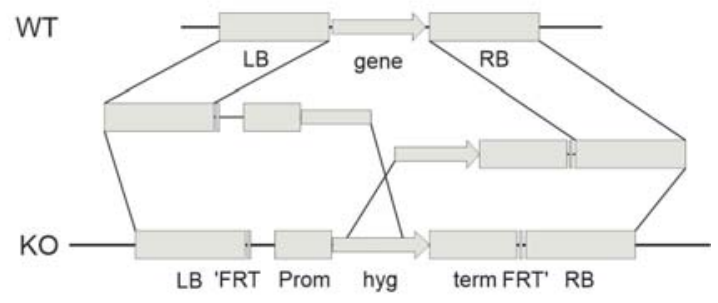

Figure 5: Genetic transformation of $M b A$. (A) Stable transformants that express cytosolic GFP could be obtained by generating protoplasts with Glucanex and ectopically integrating linear DNA-fragments into the genome via PEG-mediated transformation. (B) Overview of the split-marker approach that was used to generate deletion mutants via homologous recombination. 
Figure 6

A

U. maydis b2

$U$. maydis b1

U. hordei MAT1-1

U. hordei MAT1-2

MbA b1

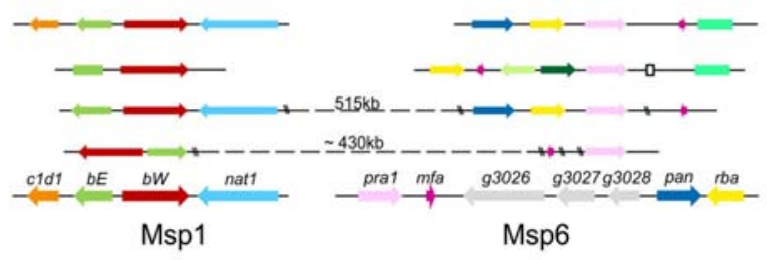

U. maydis a2

U. maydis a1

U. hordei MAT1-1

U. hordei MAT1-2

$M b A$ a1

B

C
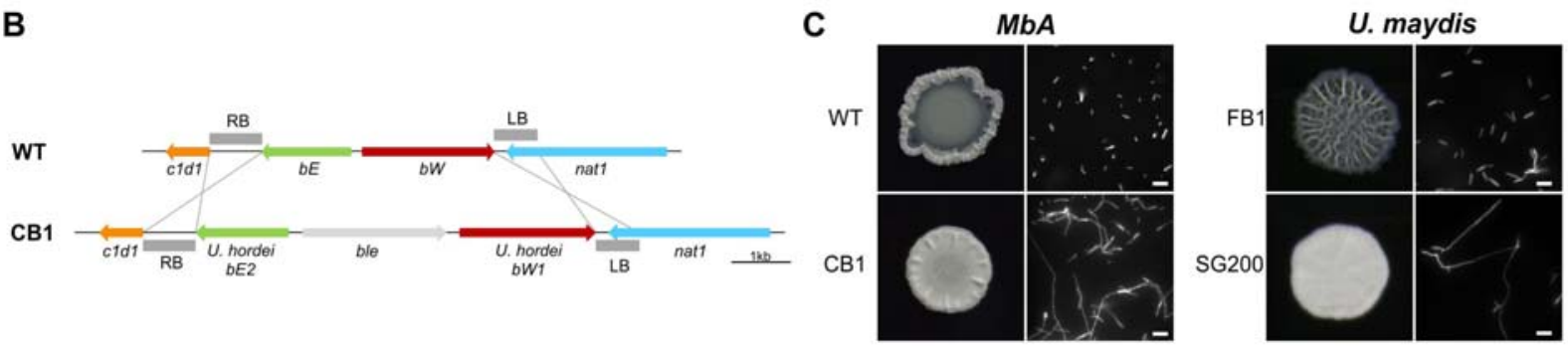

E
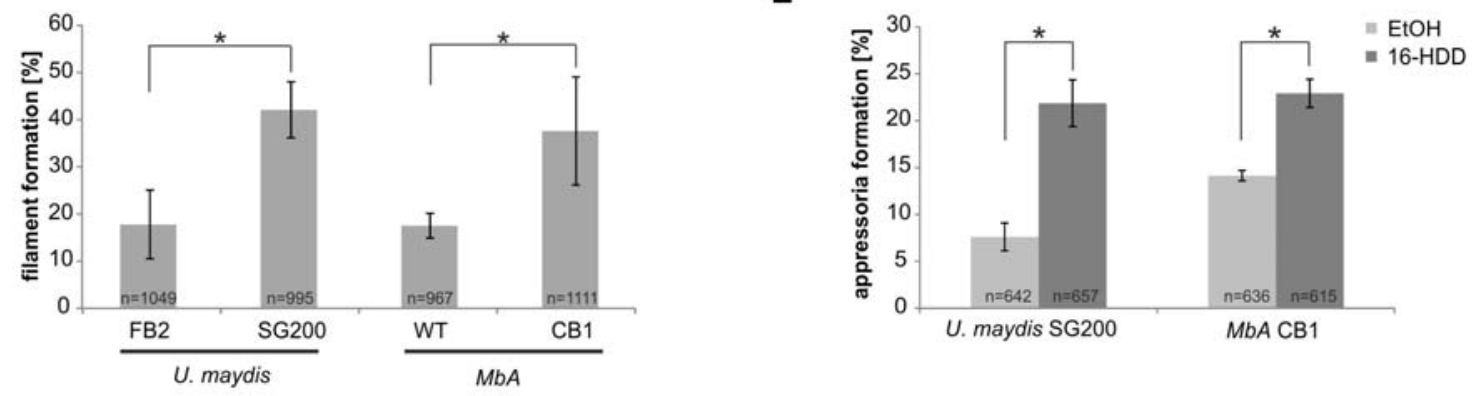

Figure 6: The self-compatible $M b A$ strain $C B 1$ (A) $M b A$ mating type genes, unlike the ones of $U$. hordei, can be found on two different chromosomes similar to the tetrapolar mating type system of $U$. maydis. (B) To generate a self-compatible strain (CB1), the bmating genes of $U$.hordei were integrated at the native $M b A b$-locus. $(C)$ Unlike the $M b A$ wild type strain (top left), strain CB1 (bottom left) shows a fluffy phenotype on charcoal plates and filamentous growth. U. maydis haploid F1 strain (top right) and self-compatible SG200 strain (bottom right) were used as negative and positive control, respectively. $(D, E)$ Induction of filamentation and appressoria formation in strain CB1 was studied in three independent experiments. For this around 1000 cells for filament formation and around 600 cells for appressoria formation were analyzed and error bars indicate standard error. After incubation on a hydrophobic surface, both, filament and appressoria formation in strain CB1, were significantly different ( ${ }^{*}$ Chi-Square Test for Independence - $\alpha=0,0001$ ) when compared to $M b A$ wild type and similar to the level of the selfcompatible $U$. maydis strain SG00. U. maydis haploid F2 strain was used as negative

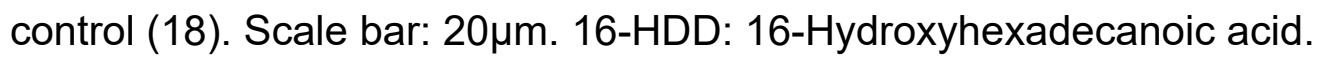




\section{Figure 7}
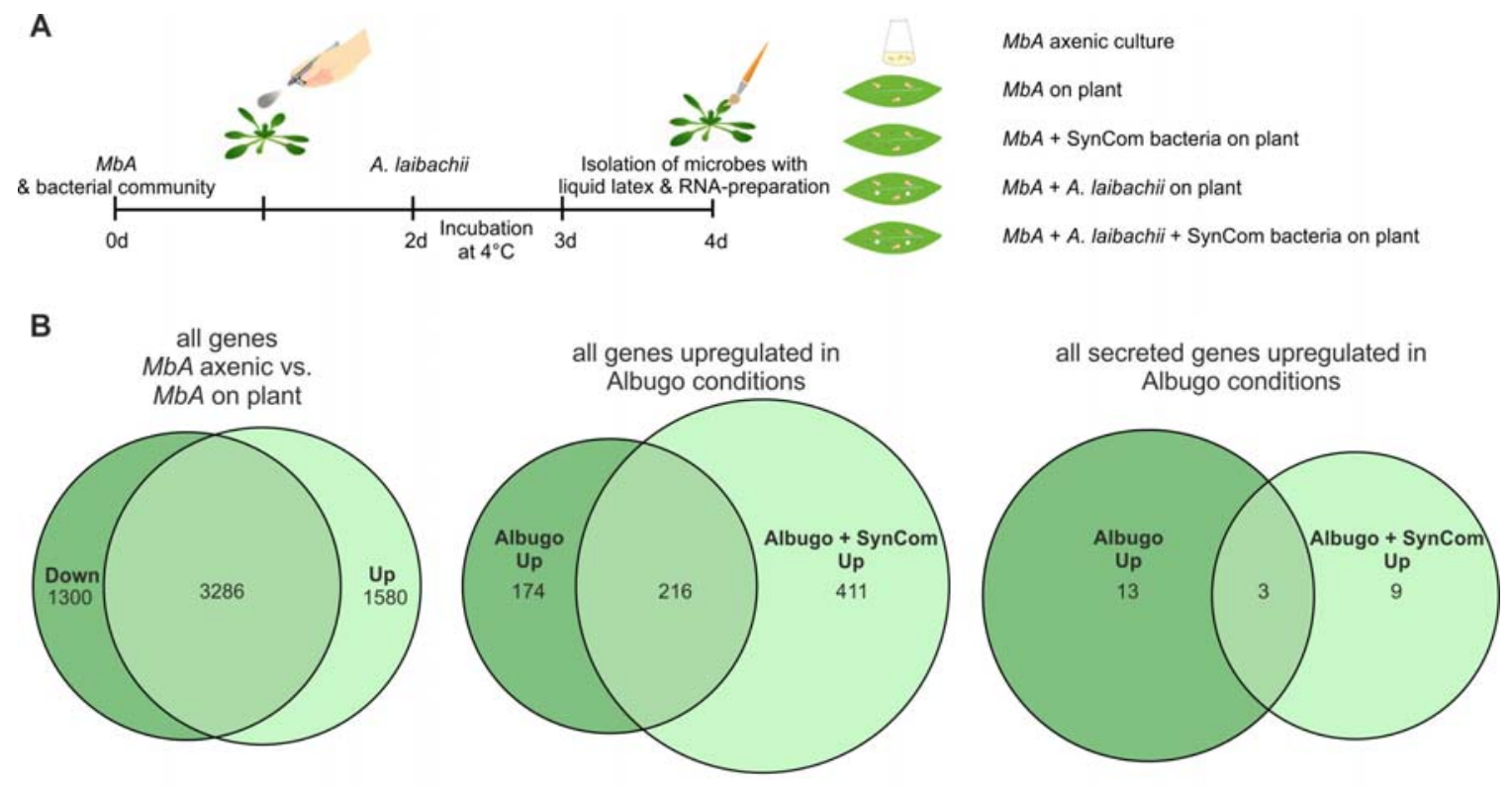

C

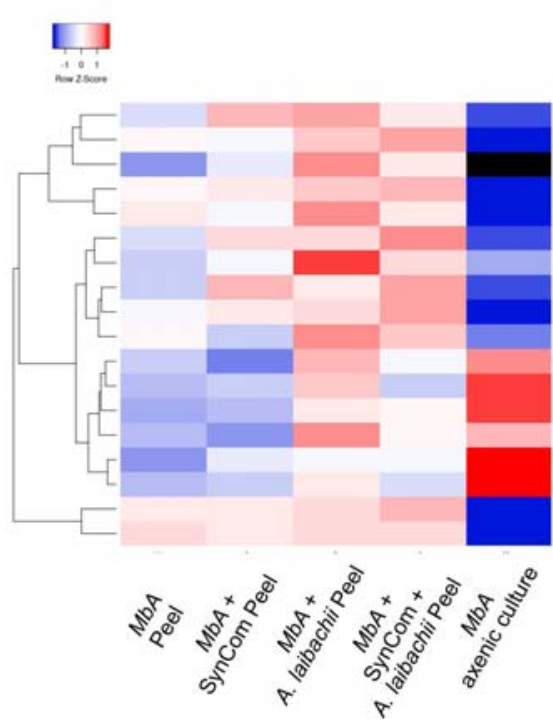

g0005 - Glycoside hydrolase (family 43)

g5755 - uncharacterized protein

g0879 - Glycoside hydrolase (family 43 -Alpha-L-Arabinofuranosidase)

g3161 - Glycoside hydrolase (family 43 - Arabinase)

g6152 - Peptidase S53 (Tripeptidyl-peptidase)

g1794 - Arginase related to CAR1

g2490 - Glycoside hydrolase (family 25 - lysozyme)

g6286 - Aromatic compound dioxygenase

g4482 - Peptidase S10 (Serine carboxypeptidase)

g5062 - FAD-binding oxidoreductase

g4707 - Glycoside hydrolase (family 31 - alpha-glucosidase)

g4583 - Glucose oxidase
g3649 - uncharacterized protein

g4658 - uncharacterized protein

g0791 - uncharacterized protein

g0254 - B2-aldehyde-forming enzyme

g3657 - uncharacterized protein

g1798 - Alkaline phosphatase

Figure 7: Transcriptome analysis of $M b A$ (A) Experimental setup used for the transcriptomic (RNA-Sequencing) analysis in MbA. (B) Venn diagrams showing differential regulated $M b A$ genes after spraying of haploid cells onto the $A$. thaliana leaf surface. A total number of 801 genes were upregulated in response to $A$. laibachii in presence and absence of bacterial SynCom. 216 of the 801 genes were upregulated in both conditions. (C) Hierarchical clustering of the 27 A. laibachii - induced MbA genes that are predicted to encode secreted proteins. Of these genes, nine were selected as candidate microbe-microbe effector genes, based on their transcriptional upregulation and prediction to encode for extracellularly localized proteins. 
Figure 8

A

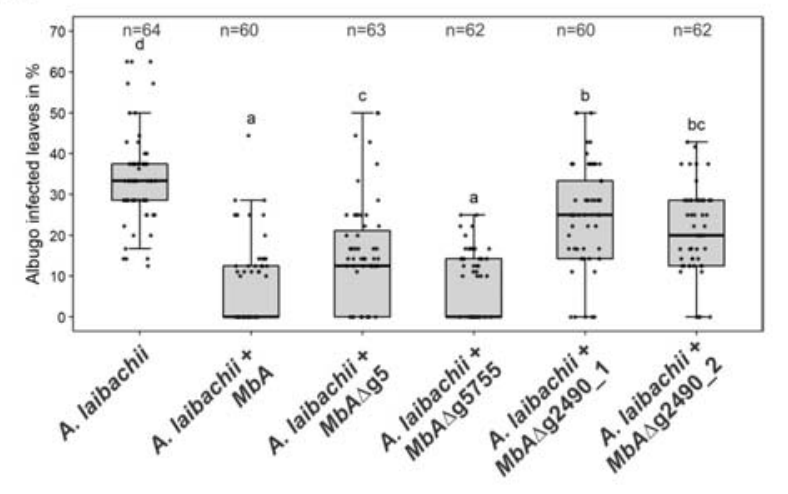

C

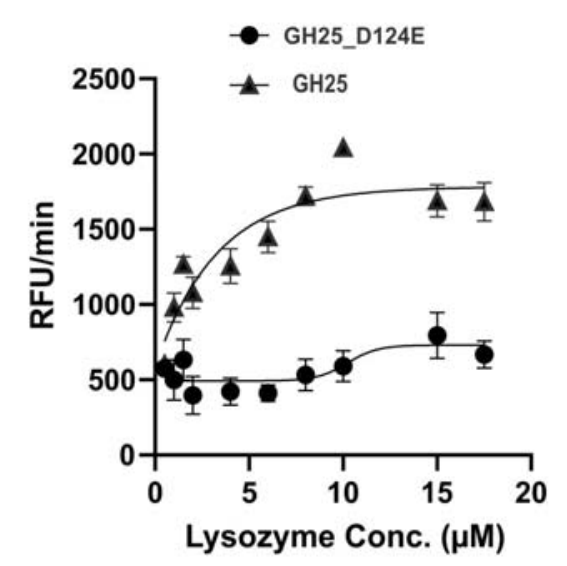

B
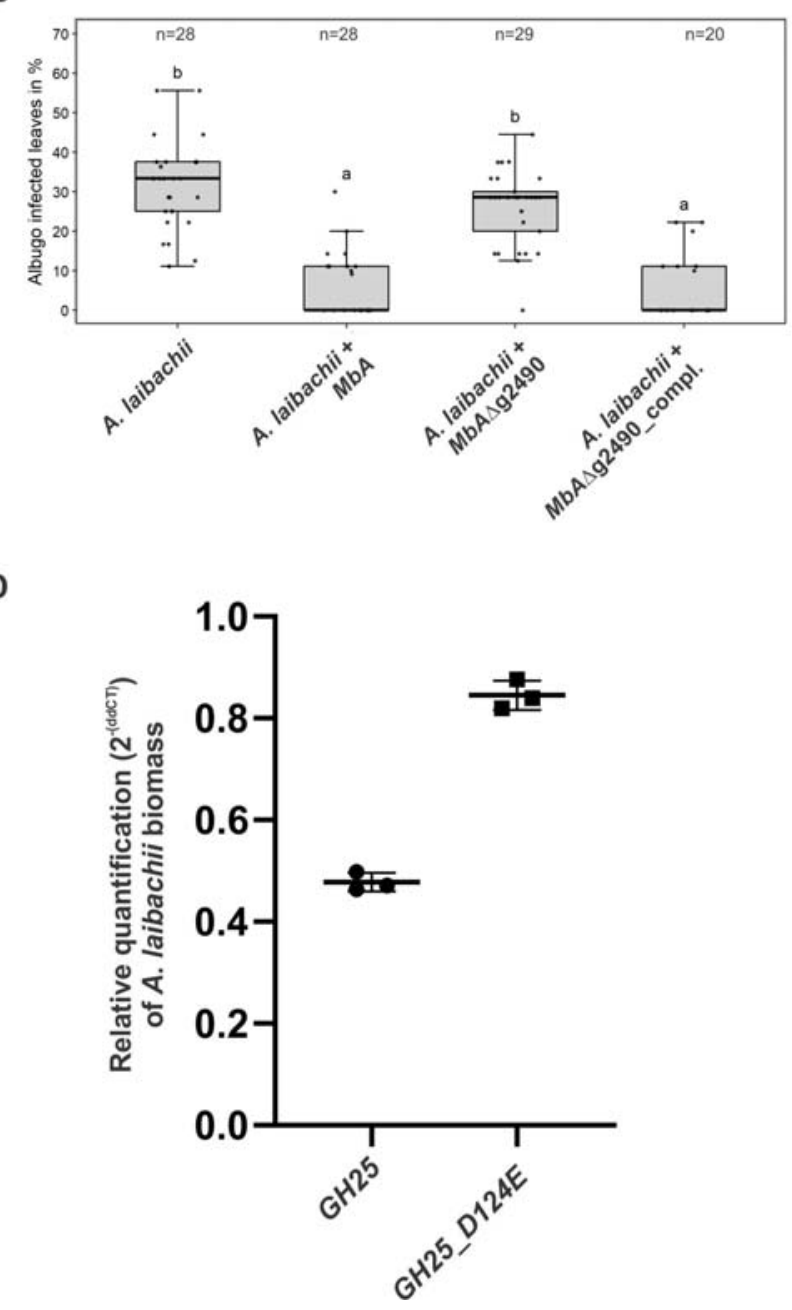

Figure 8: A reverse-genetic approach to identify the $M b A$ gene which is responsible for the suppression of $A$. laibachii infection. (A) Three candidate microbe-microbe effector genes $(g 5, g 5755 \& g 2490)$ were deleted in $M b A$ and deletion strains were individually inoculated on $A$. thaliana together with $A$. laibachii. Inoculation of two independent $g 2490$ null strains ( $\left.\Delta g 2490 \_1 ; \Delta g 2490 \_2\right)$ resulted in significant and almost complete loss the biocontrol activity of $M b A$. While deletion of g5 resulted in a marginal reduction of disease symptoms at 14 days post infection, deletion of g5755 had no effect on A. laibachii. (B) Genetic complementation of the $g 2490$ deletion restores the biocontrol activity to wild type levels. Infections in $(A)$ were performed in six, in $(B)$ in three individual replicates. In each replicate 12 plants were infected. $\mathrm{N}$ indicates the number of infected plants that were scored for symptoms. Different letters indicate significant differences ( $P$ values $<0.05$; ANOVA model for pairwise comparison with Tukey\&apos;s HSD test). (C) Detection of lysozyme. Increasing concentrations of purified MbA_GH25 and MbA_GH25(D124E) 
were incubated with the $\mathrm{DQ}$ lysozyme substrate for an hour at $37^{\circ} \mathrm{C}$. The fluorescence was recorded every minute in a fluorescence microplate reader using excitation/emission of 485/530 nm. Finally, Relative Fluorescence Unit (RFU)/ min was calculated for each concentration and plotted on the graph. Each data point represents three technical replicates and three independent biological replicates as indicated by the Standard Error Measurement (SEM) bars. An unpaired t-test was performed for the active $\mathrm{GH} 25$ and Mutant_GH25 sets giving the p-value of $<0.0001$; and R squared value of $77.24 \%$. (D) Relative quantification of $A$. laibachii biomass in response to MbA_GH25 (active and mutant) treatment via qPCR. The Oomycete internal transcribed spacer (ITS) $5.8 \mathrm{~s}$, was normalized to $A$. thaliana EF1- $\alpha$ gene to quantify the amount of $A$. laibachii DNA in the samples, ten days post infection. Then relative biomass was calculated comparing control sets (Only Albugo) with $A$. laibachii treated with $\mathrm{GH} 25$ and $A$. laibachii treated with Mutant_GH25 by ddCT method. Unpaired t-test between GH25 and Mutant_GH25 sets gave a $\mathrm{p}$-value of $<0.0001$ and an $\mathrm{R}$-squared value of $98.88 \%$. 